\title{
CLASSES OF BANACH SPACES WITH UNIQUE ISOMETRIC PREDUALS
}

\author{
LEON BROWN AND TAKASHI ITO
}

\begin{abstract}
We introduce several classes of Banach spaces for which the dual or second dual of each member has a unique isometric predual. It is shown that these classes are closed under certain operations. Examples are presented, including those which show that these classes are different.
\end{abstract}

A Banach space $Y$ is called an isometric predual, or simply a predual, of a Banach space $X$ if the dual $Y^{*}$ of $Y$ is isometrically isomorphic to $X$. A Banach space $X$ is said to have a unique (isometric) predual if $X$ has a predual and all preduals are mutually isometrically isomorphic. In general a Banach space does not have a unique predual even if it has a predual. A simple example of this is the space $l^{1}$, because $c_{0}$ and $c$ are isometric preduals of $l^{1}$ but not isometrically isomorphic. A. Grothendieck [5] first noticed that $L^{\infty}$ spaces have unique preduals, and then S. Sakai generalized this to von Neumann algebras, see p. 30 of [11]. One of the authors has shown in [6] that every quotient space of a von Neumann algebra by a $\sigma$-weakly closed subspace, as a Banach space with quotient norm, has a unique predual. Also T. Ando [1] and P. Wojtaszczyk [12] have shown that the space $H^{\infty}$ has a unique predual. Recently, we have shown in [2] that the James quasi-reflexive space and all its successive duals have unique preduals. Evidently, these are the only known nonreflexive Banach spaces with unique preduals (see Addendum).

In this paper we introduce several classes of Banach spaces for which the dual or second dual of each member has a unique isometric predual. We show that these classes are closed under certain operations. Throughout the paper, we present examples to show that these classes are different.

We use the following standard notation. We shall always regard a Banach space $X$ as a subspace of its second dual $X^{* *}$ in the canonical way. A subspace means a closed linear subspace. For a subset $A$ of a Banach space $X, A^{\perp}$ denotes the annihilator of $A$ in the dual $X^{*}$. If $A$ is a subset of a dual Banach space $X^{*}$, then $A^{\top}$ denotes the set of all elements in $X$ annihilated by $A$. For a subset $A$ of a Banach space $X,[A]$ denotes the closed linear span of $A$ in $X$, and $X=A \oplus B$ means that $X$ is the direct sum of subspaces $A$ and $B$.

If $X$ is a Banach space, then $X^{* * *}=X^{\perp} \oplus X^{*}$ where $X^{*}$ is norm 1 complemented in $X^{* * *}$. That is, the projection from $X^{* * *}$ onto 
$X^{*}$ associated with this decomposition has norm 1 . Thus a sufficient condition for $X^{*}$ to have a unique predual is that $X^{\perp}$ is the only norm 1 complement of $X^{*}$ in $X^{* * *}$. This is so because if $Y$ is a predual of $X^{*}$ then $Y$ can be identified as a subspace of $X^{* *}$ and $X^{* * *}=X^{*} \oplus Y^{\perp}$ (see Dixmier [3]). Thus $X^{\perp}=Y^{\perp}$ and $X=\left(X^{\perp}\right)^{\top}=$ $\left(Y^{\perp}\right)^{\top}=Y$. If $X^{\perp}$ is the only weak* closed norm 1 complement of $X^{*}$ in $X^{* * *}$, we say that $X$ is in class $(U)$ (All of the above examples actually have preduals which are in class $(U)$.).

In $\S 1$, we discuss unconditional basis and weak* unconditional basis with property $\left(\Delta_{1}\right)$ and property $\left(\Delta_{\infty}\right)$. These properties are used in the direct sum of Banach spaces in later sections. In $\S 2$, we introduce the classes $\left(L_{0}\right),(L),(M)$ and $(P)$, and discuss their relationships with each other and with the class $(U)$ (see Addendum). Sections 3, 4, 5 are devoted to the classes $\left(L_{0}\right)$ and $(L),(M)$, and $(P)$, respectively. In $\S 6$, we examine certain examples, namely, the James quasi-reflexive spaces, $L^{\infty}$ and $H^{\infty}$. We conclude the paper with some final remarks in $\S 6$.

1. Preliminaries. Let $\left(Z,\left\{e_{\lambda}\right\}_{\lambda \in \Lambda},\|\cdot\|\right)$ be a Banach space $Z$ with an unconditional basis $\left\{e_{\lambda}\right\}_{\lambda \in A}$ and a monotone norm $\left(\left\|\sum_{\lambda} \alpha_{\lambda} e_{\lambda}\right\| \geqq\right.$ $\left\|\sum_{\lambda} \beta_{\lambda} e_{\lambda}\right\|$ if $\left|\alpha_{\lambda}\right| \geqq\left|\beta_{\lambda}\right|$ for all $\left.\lambda \in \Lambda\right)$. We denote elements $z$ of $Z$ by $z=\left(\alpha_{\lambda}\right)$, where $\alpha_{\lambda}$ is the $e_{\lambda}$-coordinate of $z$. The dual space $\left(Z^{*},\left\{e_{\lambda}^{*}\right\}_{\lambda \in V},\|\cdot\|^{*}\right)$ has the unconditional weak ${ }^{*}$ basis $\left\{e_{\lambda}^{*}\right\}_{\lambda \in}$, which is the biorthogonal family of $\left\{e_{\lambda}\right\}_{\lambda \in A}$, and the monotone dual norm $\|\cdot\|^{*}$. We denote elements $z^{*}$ of $Z^{*}$ by $z^{*}=\left(\xi_{\lambda}\right)$, where $\xi_{\lambda}$ is the $e_{\lambda}^{*}$-coordinate of $z^{*}$. Note that if $\left\{e_{\lambda}\right\}_{\lambda \in A}$ is a shrinking basis then $\left\{e_{\lambda}^{*}\right\}_{\lambda \in}$ : actually is a basis for $Z^{*}$ (see [10] for pertinent definitions and notations).

Definition. We introduce two properties denoted by $\left(\Delta_{\infty}\right)$ and $\left(\Delta_{1}\right)$.

(1) $\left(Z,\left\{e_{\lambda}\right\}_{\lambda \in \Lambda},\|\cdot\|\right)$ has property $\left(\Delta_{\infty}\right)$ if for any $\varepsilon>0$ there is a $\rho=\rho(\varepsilon)>0$ such that

$$
\left\|\left(\alpha_{\lambda}\right)+\left(\beta_{\lambda}\right)\right\| \leqq\left\|\left(\alpha_{\lambda}\right)\right\|+\varepsilon\left\|\left(\beta_{\lambda}\right)\right\|,
$$

whenever $\left(\alpha_{\lambda}\right)$ and $\left(\beta_{\lambda}\right)$ have disjoint support (denoted by $\left(\alpha_{\lambda}\right) \perp\left(\beta_{\lambda}\right)$ ) and $\left\|\left(\alpha_{\lambda}\right)\right\| \geqq \rho\left\|\left(\beta_{\lambda}\right)\right\|$.

In a similar fashion one can define that $\left(Z^{*},\left\{e_{\lambda}^{*}\right\}_{\lambda \in \Lambda},\|\cdot\|^{*}\right)$ has property $\left(\Delta_{\infty}\right)$.

(2) $\left(Z,\left\{e_{\lambda}\right\}_{\lambda \in \Lambda},\|\cdot\|\right)$ has property $\left(\Delta_{1}\right)$ if for any $\varepsilon>0$ there is a $\gamma=\gamma(\varepsilon)>0$ such that

$$
\gamma\left\|\left(\alpha_{\lambda}\right)\right\|+\left\|\left(\beta_{\lambda}\right)\right\| \leqq(\gamma+\varepsilon)\left\|\left(\alpha_{\lambda}\right)+\left(\beta_{\lambda}\right)\right\|,
$$

whenever $\left(\alpha_{\lambda}\right) \perp\left(\beta_{\lambda}\right)$. 
In a similar fashion one can define that $\left(Z^{*},\left\{e_{\lambda}^{*}\right\}_{\lambda \in},\|\cdot\|^{*}\right)$ has property $\left(\Delta_{1}\right)$.

For $Z=c_{0}$ or $l_{p}(1 \leqq p \leqq \infty)$ one can show that $c_{0}$ and $l_{p}(1<p \leqq \infty)$ has property $\left(\Delta_{\infty}\right)$ with $\rho_{c_{0}}(\varepsilon)=\rho_{l_{\infty}}(\varepsilon)=1$ and $\rho_{l_{p}}(\varepsilon)=((q-1) / q \varepsilon)^{q-1}$, where $1 / p+1 / q=1$. Also $l_{p}(1 \leqq p<\infty)$ has property $\left(\Delta_{1}\right)$ with $\gamma_{l_{1}}(\varepsilon)=1$ and $\gamma_{l_{p}}(\varepsilon)=((p-1) / p \varepsilon)^{p-1}$. Furthermore $l_{1}$ does not have property $\left(\Delta_{\infty}\right)$ and $c_{0}$ and $l_{\infty}$ do not have property $\left(\Delta_{1}\right)$. Note also that certain Orlicz sequence spaces have property $\left(A_{1}\right)$ or property $\left(\Delta_{\infty}\right)$; see the discussion after Proposition 6 .

The duality between properties $\left(\Delta_{\infty}\right)$ and $\left(A_{1}\right)$ can be shown in a straight forward manner.

Proposition 1. Let $Z$ and $Z^{*} b e\left(Z,\left\{e_{\lambda}\right\}_{\lambda \in A},\|\cdot\|\right)$ and $\left(Z^{*},\left\{e_{\lambda}^{*}\right\}_{\lambda \in A},\|\cdot\|^{*}\right)$ respectively.

(a) $Z$ has property $\left(\Delta_{\infty}\right)$ or property $\left(\Delta_{1}\right)$ if and only if $Z^{*}$ has property $\left(\Delta_{1}\right)$ or property $\left(\Delta_{\infty}\right)$ respectively.

(b) If $Z$ has property $\left(\Delta_{\infty}\right)$ or property $\left(\Delta_{1}\right)$ then the basis $\left\{e_{\lambda}\right\}_{\lambda \in A}$ is shrinking or boundedly complete respectively. Hence if $Z$ has both property $\left(\Delta_{\infty}\right)$ and property $\left(\Delta_{1}\right), Z$ is reflexive.

(c) If $Z$ has property $\left(\Delta_{1}\right)$ then the norm of $Z$ is strictly monotone, that is, $\left\|\left(\alpha_{\lambda}\right)\right\|>\left\|\left(\beta_{\lambda}\right)\right\|$ if $\left(\left|\alpha_{\lambda}\right|\right) \supsetneqq\left(\left|\beta_{\lambda}\right|\right)$. Conversely, if $Z$ is finite dimensional and the norm is strictly monotone then $Z$ has property $\left(\Delta_{1}\right)$.

Proof. (a) Assume that $Z$ has property $\left(\Delta_{\infty}\right)$. Given $\varepsilon>0$, $\delta>0,\left(\xi_{\lambda}\right)$ and $\left(\eta_{\lambda}\right)$ in $Z^{*}$ with $\left(\xi_{\lambda}\right) \perp\left(\eta_{\lambda}\right)$, there are $\left(\alpha_{\lambda}\right)$ and $\left(\beta_{\lambda}\right)$ in $Z$ with $\left\|\left(\alpha_{\lambda}\right)\right\|=\left\|\left(\beta_{\lambda}\right)\right\|=1$ such that $\left\|\left(\xi_{\lambda}\right)\right\|^{*}-\delta \leqq \sum_{\lambda} \xi_{\lambda} \alpha_{\lambda}$ and $\left\|\left(\eta_{\lambda}\right)\right\|^{*}-\delta \leqq \sum_{\lambda} \eta_{\lambda} \beta_{\lambda}$. Furthermore, we may assume that the supports of $\left(\alpha_{\lambda}\right)$ and $\left(\beta_{\lambda}\right)$ are equal to the supports of $\left(\xi_{\lambda}\right)$ and $\left(\eta_{\lambda}\right)$ respectively. Thus

$$
\begin{aligned}
\rho(\varepsilon)\left\|\left(\xi_{\lambda}\right)\right\|^{*}+\left\|\left(\eta_{\lambda}\right)\right\|^{*} & \leqq \rho(\varepsilon) \sum_{\lambda} \xi_{\lambda} \alpha_{\lambda}+\sum_{\lambda} \eta_{\lambda} \beta_{\lambda}+(\rho(\varepsilon)+1) \delta \\
& =\sum_{\lambda}\left(\xi_{\lambda}+\eta_{\lambda}\right)\left(\rho(\varepsilon) \alpha_{\lambda}+\beta_{\lambda}\right)+(\rho(\varepsilon)+1) \delta \\
& \leqq\left\|\left(\xi_{\lambda}\right)+\left(\eta_{\lambda}\right)\right\|^{*}\left\|\rho(\varepsilon)\left(\alpha_{\lambda}\right)+\left(\beta_{\lambda}\right)\right\|+(\rho(\varepsilon)+1) \delta \\
& \leqq\left\|\left(\xi_{\lambda}\right)+\left(\eta_{\lambda}\right)\right\|^{*}(\rho(\varepsilon)+\varepsilon)+(\rho(\varepsilon)+1) \delta .
\end{aligned}
$$

Hence we can conclude that $\rho(\varepsilon)\left\|\left(\xi_{\lambda}\right)\right\|^{*}+\left\|\left(\eta_{\lambda}\right)\right\|^{*} \leqq(\rho(\varepsilon)+\varepsilon)\left\|\left(\xi_{\lambda}\right)+\left(\eta_{\lambda}\right)\right\|^{*}$ which means that $Z^{*}$ has property $\left(\Delta_{1}\right)$ with $\gamma(\varepsilon)=\rho(\varepsilon)$ for all $\varepsilon>0$.

Conversely, suppose that $Z^{*}$ has property $\left(\Delta_{1}\right)$ and we are given $\varepsilon>0,\left(\alpha_{\lambda}\right)$ and $\left(\beta_{\lambda}\right)$ in $Z$ with $\left(\alpha_{\lambda}\right) \perp\left(\beta_{\lambda}\right)$. Choose $\left(\xi_{\lambda}\right)$ in $Z^{*}$ so that $\left\|\left(\xi_{\lambda}\right)\right\|^{*}=1$ and $\sum_{\lambda} \xi_{\lambda}\left(\alpha_{\lambda}+\beta_{\lambda}\right)=\left\|\left(\alpha_{\lambda}\right)+\left(\beta_{\lambda}\right)\right\|$. Setting $\xi_{\lambda}^{\prime}=\xi_{\lambda}\left(\xi_{\lambda}^{\prime \prime}=\xi_{\lambda}\right)$ if $\lambda$ is in the support of $\left(\alpha_{\lambda}\right)\left(\left(\beta_{\lambda}\right)\right)$, one has $\left(\xi_{\lambda}\right)=\left(\xi_{\lambda}^{\prime}\right)+\left(\xi_{\lambda}^{\prime \prime}\right)$ with 
$\left(\xi_{\lambda}^{\prime}\right) \perp\left(\xi_{\lambda}^{\prime \prime}\right)$ and $\left\|\left(\alpha_{\lambda}\right)+\left(\beta_{\lambda}\right)\right\|=\sum_{\lambda}\left(\xi_{\lambda}^{\prime}+\xi_{\lambda}^{\prime \prime}\right)\left(\alpha_{\lambda}+\beta_{\lambda}\right)=\sum_{\lambda} \xi_{\lambda}^{\prime} \alpha_{\lambda}+\sum_{\lambda} \xi_{\lambda}^{\prime \prime} \beta_{\lambda} \leqq$ $\left\|\left(\alpha_{\lambda}\right)\right\|\left\|\left(\xi_{\lambda}^{\prime}\right)\right\|^{*}+\left\|\left(\beta_{\lambda}\right)\right\|\left\|\left(\xi_{\lambda}^{\prime \prime}\right)\right\|^{*}$. If $\left\|\left(\alpha_{\lambda}\right)\right\| \geqq \gamma(\varepsilon)\left\|\left(\beta_{\lambda}\right)\right\|$, we have

$$
\begin{aligned}
\left\|\left(\alpha_{\lambda}\right)\right\|\left\|\left(\xi_{\lambda}^{\prime}\right)\right\|^{*}+\left\|\left(\beta_{\lambda}\right)\right\|\left\|\left(\xi_{\lambda}^{\prime \prime}\right)\right\|^{*} & \leqq\left\|\left(\beta_{\lambda}\right)\right\|\left[\frac{\left\|\left(\alpha_{\lambda}\right)\right\|}{\left\|\left(\beta_{\lambda}\right)\right\|}\left\|\left(\xi_{\lambda}^{\prime}\right)\right\|^{*}+\left\|\left(\xi_{\lambda}^{\prime \prime}\right)\right\|^{*}\right] \\
& \leqq\left\|\left(\beta_{\lambda}\right)\right\|\left[\frac{\left\|\left(\alpha_{\lambda}\right)\right\|}{\left\|\left(\beta_{\lambda}\right)\right\|}+\varepsilon\right]\left\|\left(\xi_{\lambda}^{\prime}\right)+\left(\xi_{\lambda}^{\prime \prime}\right)\right\|^{*} \\
& =\left\|\left(\alpha_{\lambda}\right)\right\|+\varepsilon\left\|\left(\beta_{\lambda}\right)\right\| .
\end{aligned}
$$

Thus $Z$ has property $\left(\Delta_{\infty}\right)$ with $\rho(\varepsilon)=\gamma(\varepsilon)$ for all $\varepsilon>0$.

In a similar fashion one can show that $Z$ has property $\left(\Delta_{1}\right)$ if and only if $Z^{*}$ has property $\left(A_{\infty}\right)$. We omit the details of this proof.

(b) Assume that $Z$ has property $\left(A_{\infty}\right)$. By part (a) $Z^{*}$ has property $\left(A_{1}\right)$. Given $\left(\xi_{\lambda}\right)$ in $Z^{*}$ with $\left\|\left(\xi_{2}\right)\right\|^{*}=1$ and $\varepsilon>0$ there is a $\gamma=\gamma(\varepsilon)>0$ so that for all finite subsets $H$ of $\Lambda \gamma\left\|\left.\left(\xi_{2}\right)\right|_{H}\right\|^{*}+$ $\left\|\left.\left(\xi_{\lambda}\right)\right|_{H^{c}}\right\|^{*} \leqq \gamma+\varepsilon$ or $\left\|\left.\left(\xi_{\lambda}\right)\right|_{H^{c}}\right\|^{*} \leqq \gamma\left(1-\left\|\left.\left(\xi_{\lambda}\right)\right|_{H}\right\|^{*}\right)+\varepsilon$. Since $\left\{e_{\lambda}^{*}\right\}_{\lambda \in A}$ is a weak* basis for $Z^{*}$ we have $1=\left\|\left(\xi_{\lambda}\right)\right\|^{*}=\sup _{H}\left\|\left.\left(\xi_{\lambda}\right)\right|_{H}\right\|^{*}$. Choosing a finite subset $H$ of $A$ so that $\gamma\left(1-\left\|\left.\left(\xi_{2}\right)\right|_{H}\right\|^{*}\right)<\varepsilon$, we have $\left\|\left.\left(\xi_{\lambda}\right)\right|_{H^{c}}\right\|^{*}<2 \varepsilon$. Thus the base $\left\{e_{\lambda}\right\}_{\lambda \in A}$ is shrinking.

Assume that $Z$ has property $\left(A_{1}\right)$. Suppose we have a function $\varphi$ on $\Lambda$ with $\sup _{H}\left\|\left.\varphi\right|_{I I}\right\|<+\infty$, where $H$ is a finite subset of $\Lambda$. We need to show that there is an element $\left(\alpha_{\lambda}\right)$ in $Z$ such that $\varphi(\lambda)=\alpha_{\lambda}$ for all $\lambda \in \Lambda$. To see this, it is enough to show that for any $\varepsilon>0$ there is a finite subset $H_{\varepsilon}$ of $\Lambda$ such that $\left\|\left.\varphi\right|_{F}\right\|<\varepsilon$ for all finite subsets $F \subset H_{\varepsilon}^{c}$. Without loss of generality, we may assume $\sup _{I I}\left\|\left.\varphi\right|_{H}\right\|=1$. Given $\varepsilon>0$ there is $\gamma=\gamma(\varepsilon)>0$ such that

$$
\gamma\left\|\left.\varphi\right|_{H}\right\|+\left\|\left.\varphi\right|_{F}\right\| \leqq(\gamma+\varepsilon)\left\|\left.\varphi\right|_{H \cup F}\right\| \leqq \gamma+\varepsilon
$$

for any finite subsets $H$ and $F$ with $H \cap F=\varnothing$. Thus, choosing a finite subset $H_{\varepsilon}$ of $\Lambda$ so that $\gamma\left(1-\left\|\left.\varphi\right|_{H}\right\|\right)<\varepsilon$, we have $\left\|\left.\varphi\right|_{F}\right\|<2 \varepsilon$ for all finite subsets $F \subset H_{\varepsilon}^{c}$.

(c) Suppose the norm $\|\cdot\|$ of $Z$ is not strictly monotone. Then there is a $\left(\alpha_{\lambda}\right)$ in $Z$, a $\lambda_{0}$ in $\Lambda$ and a $\delta$ with $0 \leqq \delta<1$ such that $e_{\lambda_{0}} \perp\left(\alpha_{\lambda}\right)$ and $\left\|e_{\lambda_{0}}+\left(\alpha_{\lambda}\right)\right\|=\left\|\delta e_{\lambda_{0}}+\left(\alpha_{\lambda}\right)\right\|$. Since the increasing convex function $f(t)=\left\|t e_{0}+\left(\alpha_{2}\right)\right\|$ for $t \geqq 0$ is constant for $\delta \leqq t \leqq 1, f$ must be constant for $0 \leqq t \leqq 1$ and one has $\left\|e_{\lambda_{0}}+\left(\alpha_{2}\right)\right\|=\left\|\left(\alpha_{2}\right)\right\|$. Choose $\varepsilon>0$ so that $\left\|e_{\lambda_{0}}\right\|>\varepsilon\left\|\left(\alpha_{\lambda}\right)\right\|$. Thus we have, for all $\gamma>0$, $\gamma\left\|\left(\alpha_{\lambda}\right)\right\|+\left\|e_{\lambda_{0}}\right\|>(\gamma+\varepsilon)\left\|e_{\lambda_{0}}+\left(\alpha_{\lambda}\right)\right\|$ which implies that $Z$ does not have property $\left(\Delta_{1}\right)$.

Conversely suppose that $Z$ is finite dimensional and the norm $\|\cdot\|$ is a strictly monotone norm without having property $\left(\Delta_{1}\right)$. If in the inequality defining property $\left(\Delta_{1}\right)$ one replaces $\left|\alpha_{\lambda}\right|+\left|\beta_{\lambda}\right|$ by $\alpha_{\lambda}$ and $\left|\alpha_{\lambda}\right|$ by $\beta_{\lambda}$ one sees that for some $\varepsilon_{0}>0$ there are $\left(\alpha_{\lambda}^{(n)}\right)$ and $\left(\beta_{\lambda}^{(n)}\right)$ in $Z$ with $\left\|\left(\alpha_{\lambda}^{(n)}\right)\right\|=1,\left(\alpha_{\lambda}^{(n)}\right) \geqq\left(\beta_{\lambda}^{(n)}\right) \geqq 0$ and $2 \geqq\left\|\left(\alpha_{\lambda}^{(n)}\right)-\left(\beta_{\lambda}^{(n)}\right)\right\| \geqq$ 
$n\left[1-\left\|\left(\beta_{\lambda}^{(n)}\right)\right\|\right]+\varepsilon_{0}$ for all $n=1,2, \cdots$. Since $Z$ is finite dimensional we may choose accumulation points $\left(\alpha_{\lambda}\right)$ and $\left(\beta_{\lambda}\right)$ of $\left\{\left(\alpha_{\lambda}^{(n)}\right) ; n=1,2, \cdots\right\}$ and $\left\{\left(\beta_{\lambda}^{(n)}\right) ; n=1,2, \cdots\right\}$ respectively. Then we have $\left(\alpha_{\lambda}\right) \geqq\left(\beta_{\lambda}\right) \geqq 0$ and $\left\|\left(\alpha_{\lambda}\right)\right\|=\left\|\left(\beta_{\lambda}\right)\right\|=1$ and $\|\left(\alpha_{\lambda}\right)-\left(\beta_{\lambda}\right) \mid \geqq \varepsilon_{0}>0$, which contradicts the assumption that the norm is strictly monotone. The proof is complete.

Given $\left(Z,\left\{e_{\lambda}\right\}_{\lambda \in \Lambda},\|\cdot\|\right)$ and a family of Banach spaces $X_{\lambda}(\lambda \in \Lambda)$, the $Z$-direct sum of $\left\{X_{\lambda}\right\}_{\lambda \in \Lambda}$, denoted by $\left(\sum_{\lambda} X_{\lambda}\right)_{z}$, is defined to be the Banach space consisting of $x=\left(x_{\lambda}\right), x_{\lambda} \in X_{\lambda}(\lambda \in \Lambda)$ such that $\left(\left\|x_{\lambda}\right\|_{X_{\lambda}}\right) \in Z$ with the norm of $x,\|x\|=\left\|\left(\left\|x_{\lambda}\right\|_{X_{\lambda}}\right)\right\|_{z}$. For the dual $\left(Z^{*},\left\{e_{\lambda}^{*}\right\}_{\lambda \in \Lambda},\|\cdot\|^{*}\right)$ of $\left(Z,\left\{e_{\lambda}\right\}_{\lambda \in \Lambda},\|\cdot\|\right)$ one can define the $Z^{*}$-direct sum of $\left\{X_{\lambda}\right\}_{\lambda \in \Lambda}$ in a similar manner, denoted by $\left(\sum_{\lambda} X_{\lambda}\right)_{Z^{*}}$.

Throughout this paper except for $\S 6, Z$ stands for $\left(Z,\left\{e_{\lambda}\right\}_{\lambda \in \Lambda},\|\cdot\|\right)$ with an unconditional base $\left\{e_{\lambda}\right\}_{\lambda \in A}$ and a monotone norm $\|\cdot\|, Z^{*}$ stands for $\left(Z^{*},\left\{e_{\lambda}^{*}\right\}_{\lambda \in \Lambda},\|\cdot\|^{*}\right)$ and $\left.Z\right|_{F}$ or $\left.Z^{*}\right|_{F}$ stands for the restriction of $Z$ or $Z^{*}$ onto a subset $F$ of $\Lambda$.

2. Definition of the classes. We first introduce the following class $\left(L_{0}\right)$ of Banach spaces.

Definition of $\left(L_{0}\right)$. A Banach space $X$ is in class $\left(L_{0}\right)$ if the canonical decomposition $X^{* * *}=X^{*} \oplus X^{\perp}$ is of $l_{1}$-type, namely, we have for all $x^{*} \in X^{*}$ and $f \in X^{\perp}$

$$
\left\|x^{*}+f\right\|=\left\|x^{*}\right\|+\|f\| \text {. }
$$

A typical example of a nonreflexive space in class $\left(L_{0}\right)$ is $c_{0}$. It is not too difficult to see this, because we can identify $c_{0}^{* * *}$ as all regular Borel measures on the Stone-Čech compactification $\beta N$ of all positive integers ' $N$. Then $c_{0}^{*}$ is the subspace of all measures supported on $N$ and $c_{0}^{1}$ is the subspace of all measures supported on $\beta N \backslash N$. Another interesting example of this class is the Banach space of all compact operators on a Hilbert space. We will discuss this example later; see Proposition 5.

A dual characterization of $\left(L_{0}\right)$ is the following.

Proposition 2. A Banach space $X$ is in class $\left(L_{0}\right)$ if and only if in the canonical decomposition $X^{(4)}=X^{* *} \oplus\left(X^{*}\right)^{\perp}$ we have for all $x \in X \subset X^{* *}$ and $\varphi \in\left(X^{*}\right)^{\perp}$

$$
\|x+\varphi\|=\operatorname{Max}\{\|x\|,\|\varphi\|\} .
$$

Proof. Assume that $X$ is in class $\left(L_{0}\right)$. For $x \in X \subset X^{* *}, \phi \in$ $\left(X^{*}\right)^{\perp}, x^{*} \in X^{*}$ and $f \in X^{\perp}$ we have $\left|\left\langle x^{*}+f, x+\varphi\right\rangle\right| \leqq\left|\left\langle x^{*}, x\right\rangle\right|+$ 
$|\langle f, \varphi\rangle| \leqq\left\|x^{*}\right\|\|x\|+\|f\|\|\varphi\| \leqq\left(\left\|x^{*}\right\|+\|f\|\right) \operatorname{Max}\{\|x\|,\|\varphi\|\}=$ $\left\|x^{*}+f\right\| \operatorname{Max}\{\|x\|,\|\varphi\|\} ;$ hence $\|x+\varphi\| \leqq \operatorname{Max}\{\|x\|,\|\varphi\|\}$. We wish to show the reverse inequality; $\|x+\varphi\| \geqq \operatorname{Max}\{\|x\|,\|\varphi\|\}$. It is clear that $\|x+\varphi\| \geqq\|x\|$. Observe that $\left(X^{*}\right)^{\perp}$ is the dual of $X^{* * *} / X^{*}$ and that $X^{* * *} / X^{*}$ is isometrically isomorphic to $X^{\perp}$ in the canonical way, because $X$ is in class $\left(L_{0}\right)$. Hence $\|\varphi\|=\sup \left\{\mid\langle f, \varphi\rangle \| f \in X^{\perp}\right.$, $\|f\| \leqq 1\} \sup \left\{\mid\langle f, x+\varphi\rangle\left\|f \in X^{\perp},\right\| f \| \leqq 1\right\} \leqq\|x+\varphi\|$. Thus we have $\|x+\varphi\| \geqq \operatorname{Max}\{\|x\|,\|\varphi\|\}$.

Conversely assume that $\|x+\varphi\|=\operatorname{Max}\{\|x\|,\|\varphi\|\}$ for all $x \in$ $X \subset X^{* * *}$ and $\varphi \in\left(X^{*}\right)^{\perp}$. For $x^{*} \in X^{*}$ and $f \in X^{\perp}$ we have $\left\|x^{*}+f\right\|=$ $\sup \left\{\left|\left\langle x^{*}+f, x+\varphi\right\rangle\right|=\left|\left\langle x^{*}, x\right\rangle+\langle f, \varphi\rangle\right| \mid x \in X\right.$ with $\|x\| \leqq 1, \varphi \in\left(X^{*}\right)^{\perp}$ with $\|\varphi\| \leqq 1\}=\sup \left\{\mid\left\langle x^{*}, x\right\rangle\|x \in X\|, x \| \leqq 1\right\}+\sup \left\{\mid\langle f, \varphi\rangle \| \varphi \in\left(X^{*}\right)^{\perp}\right.$, $\|\varphi\| \leqq 1\}=\left\|x^{*}\right\|+\left\|f+X^{*}\right\|$. Thus we have $\left\|x^{*}+f\right\| \geqq\left\|x^{*}\right\|+$ $\left\|f+X^{*}\right\|$ for all $x^{*} \in X^{*}$ and $f \in X^{\perp}$. We conclude the proof by showing $\left\|f+X^{*}\right\|=\|f\|$. Given $\varepsilon>0$, choose $y^{*} \in X^{*}$ so that $\left\|f+y^{*}\right\|<\left\|f+X^{*}\right\|+\varepsilon$, then the inequality proved just above gives us $\left\|y^{*}\right\|+\left\|f+X^{*}\right\| \leqq\left\|f+y^{*}\right\|<\left\|f+X^{*}\right\|+\varepsilon$. Thus we have $\left\|y^{*}\right\|<\varepsilon$ and $\left\|f+X^{*}\right\|>\left\|f+y^{*}\right\|-\varepsilon \geqq\|f\|-\left\|y^{*}\right\|-\varepsilon>$ $\|f\|-2 \varepsilon$. This completes the proof.

One easily shows the following result.

Corollary. If $X$ and $X^{*}$ are in class $\left(L_{0}\right)$ then $X$ is reflexive.

One can consider the following property which generalizes $\left(L_{0}\right)$.

Definition of $(L)$. A Banach space $X$ is in class $(L)$ if in the canonical decomposition $X^{* * *}=X^{*} \oplus X^{\perp}$, for any $\varepsilon>0$ there is a $\gamma=\gamma(\varepsilon)>0$ such that for all $x^{*} \in X^{*}$ and $f \in X^{\perp}$

$$
\|f\| \leqq \gamma\left(\left\|x^{*}+f\right\|-\left\|x^{*}\right\|\right)+\varepsilon\left\|x^{*}+f\right\| .
$$

The following equivalent formulation of $(L)$ may be more understandable. For any $\varepsilon>0$ there is a $\delta=\delta(\varepsilon)>0$ such that $\|f\|<\varepsilon$ whenever we have $\left\|x^{*}+f\right\|=1$ and $1-\left\|x^{*}\right\|<\delta$ for $x^{*} \in X^{*}$ and $f \in X^{\perp}$.

The simplest example of a nonreflexive Banach space in this class $(L)$ may be the $l_{p}$-direct sum of $c_{0},\left(\sum c_{0}\right)_{l_{p}}$, for $1<p<\infty$. Also Banach spaces $Z$ with property $\left(\Delta_{\infty}\right)$ are in class $(L)$. These facts will be shown in a more general setting later; see Theorem 2 and Proposition 6.

One can give the dual property of $(L)$.

Proposition 3. A Banach space $X$ is in class $(L)$ if and only 
if in the canonical decomposition $X^{(4)}=X^{* *} \oplus\left(X^{*}\right)^{\perp}$, for any $\varepsilon>0$ there is a $\rho=\rho(\varepsilon)>0$ such that we have

$$
\|x+\varphi\| \leqq\|x\|+\varepsilon\|\varphi\|
$$

whenever $\|x\| \geqq \rho\|\varphi\|$ where $x \in X \subset X^{* *}$ and $\varphi \in\left(X^{*}\right)^{\perp}$.

Proof. Assume that $X$ is in class $(L)$. Then we have for all $x^{*} \in X^{*}, f \in X^{\perp}$ and $t>\gamma(\varepsilon),\|f\| \leqq \gamma\left(\left\|x^{*}+f\right\|-\left\|x^{*}\right\|\right)+\varepsilon\left\|x^{*}+f\right\| \leqq$ $t\left(\left\|x^{*}+f\right\|-\left\|x^{*}\right\|\right)+\varepsilon\left\|x^{*}+f\right\|$ and thus $\left\|x^{*}+f\right\| \geqq(t /(t+\varepsilon))\left\|x^{*}\right\|+$ $(1 /(t+\varepsilon))\|f\|$. For $x \in X \subset X^{* *}$ and $\varphi \in\left(X^{*}\right)^{\perp}$ with $\|x\| /\|\varphi\|=t>\gamma(\varepsilon)$ $\left|\left\langle x^{*}+f, x+\varphi\right\rangle\right|=\left|\left\langle x^{*}, x\right\rangle+(f, \varphi)\right| \leqq\left\|x^{*}\right\|\|x\|+\|f\|\|\varphi\|=t\|\varphi\|\left\|x^{*}\right\|+$ $\|\varphi\|\|f\|=\|\varphi\|(t+\varepsilon)\left[(t /(t+\varepsilon))\left\|x^{*}\right\|+(1 /(t+\varepsilon))\|f\|\right] \leqq\|\varphi\|(t+\varepsilon)\left\|x^{*}+f\right\|=$ $(\|x\|+\varepsilon\|\varphi\|)\left\|x^{*}+f\right\|$. Thus by setting $\rho=\rho(\varepsilon)=\gamma(\varepsilon)$, we have $\|x+\varphi\| \leqq\|x\|+\varepsilon\|\varphi\|$ if $\|x\|>\rho\|\varphi\|$.

Conversely, assume that $X$ satisfies the property stated in the proposition. For given $\varepsilon>0, x^{*} \in X^{*}, f \in X^{\perp}, x \in X \subset X^{* *}$ with $\|x\|=1$, $\phi \in\left(X^{*}\right)^{\perp}$ with $\|\varphi\|=1$ and $\rho=\rho(\varepsilon / 2)$ we have $\left|\rho\left\langle x^{*}, x\right\rangle+\langle f, \varphi\rangle\right|=$ $\left|\left\langle x^{*}+f, \rho x+\varphi\right\rangle\right| \leqq\left\|x^{*}+f\right\|\|\rho x+\varphi\| \leqq\left\|x^{*}+f\right\| \rho+(\varepsilon / 2)$. By taking supremum over all $x \in X$ with $\|x\|=1$ and all $\varphi \in\left(X^{*}\right)^{\perp}$ with $\|\varphi\|=1$, we have $\rho\left\|x^{*}\right\|+\left\|f+X^{*}\right\| \leqq\left\|x^{*}+f\right\|(\rho+\varepsilon / 2)$. Hence $(1 / 2)\|f\| \leqq\left\|f+X^{*}\right\| \leqq \rho\left(\left\|x^{*}+f\right\|-\left\|x^{*}\right\|\right)+(\varepsilon / 2)\left\|x^{*}+f\right\|$. Thus we have shown $\|f\| \leqq \gamma\left(\left\|x^{*}+f\right\|-\left\|x^{*}\right\|\right)+\varepsilon\left\|x^{*}+f\right\|$, where $\gamma=$ $\gamma(\varepsilon)=2 \rho(\varepsilon / 2)$. This completes the proof.

Proposition 3 suggests the following definition.

Definition of $(M)$. A Banach space $X$ is in class $(M)$ if there exists a subspace $Y$ of $X^{*}$ which norms $X$ such that given $\varepsilon>0$ there is a $\rho=\rho(\varepsilon)>0$ such that in the canonical decomposition $X^{* * *}=X^{*} \oplus X^{\perp}$, we have

$$
\left\|y^{*}+f\right\| \leqq\left\|y^{*}\right\|+\varepsilon\|f\|
$$

if $\left\|y^{*}\right\| \geqq \rho\|f\|$ where $y^{*} \in Y$ and $f \in X^{\perp}$.

Note that if $X$ is nonreflexive then $Y$ must be a proper subspace of $X^{*}$ because if $Y=X^{*}$, then we can choose a net $y_{\lambda}^{*}(\lambda \in \Lambda)$ in $Y$ such that $\left\|y_{\lambda}^{*}\right\|=\|f\|>0$ and $y_{\lambda}^{*} \stackrel{w^{*}}{\longrightarrow} f$ in $X^{* * *}$. Then we have $(\rho+1)\|f\|=\|\rho f+f\| \leqq \sup _{\lambda}\left\|\rho y_{\lambda}^{*}+f\right\| \leqq \sup _{\lambda}\left(\left\|\rho y_{\lambda}^{*}\right\|+\varepsilon\|f\|\right)=$ $(\rho+\varepsilon)\|f\|$ and arrive at a contradiction. Also note that Proposition 3 can be restated to say that a Banach space $X$ is in class $(L)$ if and only if $X^{*}$ is in class $(M)$ with the norming subspace $Y$ of $X^{* *}$ chosen to be $X$. 
Definition of $(P)$. A Banach space $X$ is in class $(P)$ if in the canonical decomposition $X^{* * *}=X^{*} \oplus X^{\perp}$,

(P) $\quad\left\|x^{* * *}+x^{*}\right\| \geqq\left\|x^{*}\right\|$ for all $x^{*} \in X^{*}$ implies $x^{* * *} \in X^{\perp}$.

This property is equivalent to saying that whenever we have a norm 1 projection $P$ onto $X^{*}$ from a subspace $Y$ of $X^{* * *}$ then $P$ must be the restriction onto $Y$ of the canonical projection onto $X^{*}$ from $X^{* * *}$. Hence if $X$ is in class $(P)$, then $X^{\perp}$ in the only norm 1 complement of $X^{*}$ in $X^{* * *}$, and hence $X$ is in class $(U)$. An example of nonreflexive Banach spaces in class $(P)$ is the $l_{p}$-direct sum of $\left(\sum l_{1}\right)_{l_{p_{n}}} ;\left(\sum_{n}\left(\sum l_{1}\right)_{l_{p_{n}}}\right) l_{l_{p}}$ where $1 \leqq p_{n}<\infty(n=1,2, \cdots)$ and $1 \leqq p<\infty$; see Theorem 4. Other interesting examples are all successive duals of the James' quasi-reflexive space $J$. This fact has been proved in [2].

Proposition 4. The class $(M)$ is a subclass of $(P)$.

Proof. Suppose a Banach space $X$ is in class $(M)$ and there is $x_{0}^{* * *} \in X^{* * *}$ such that $\left\|x_{0}^{* * *}+x^{*}\right\| \geqq\left\|x^{*}\right\|$ for all $x^{*} \in X^{*}$. We claim that $x_{0}^{* * *}$ is in $X^{\perp}$. From the canonical decomposition we have $x_{0}^{* * *}=-x_{0}^{*}-f_{0}$ where $x_{0}^{*} \in X^{*}$ and $f_{0} \in X^{\perp}$. Thus we have

$$
\left\|f_{0}+x^{*}\right\| \geqq\left\|x_{0}^{*}+x^{*}\right\| \quad \text { for all } x^{*} \in X^{*} .
$$

Since $X$ is in class $(M)$ there is a norming subspace $Y \subset X^{*}$ such that given $\varepsilon>0$ there is a $\rho=\rho(\varepsilon)>0$ such that if $y^{*} \in Y$ and $\left\|y^{*}\right\| \geqq \rho\left\|f_{0}\right\|$ then

$$
\left\|y^{*}+f_{0}\right\| \leqq\left\|y^{*}\right\|+\varepsilon\left\|f_{0}\right\| .
$$

If $x_{0}^{*} \neq 0$ then we choose a $K$ so that $K\left\|x_{0}^{*}\right\|>\rho\left\|f_{0}\right\|$. The unit ball of $Y$ is weak* dense in the unit ball of $X^{*}$, because $Y$ norms $X$. There is a net $y_{\lambda}^{*}(\lambda \in \Lambda)$ in $Y$ with $\left\|y_{\lambda}^{*}\right\|=K\left\|x_{0}^{*}\right\|$ and $y_{\lambda}^{*} \stackrel{w^{*}}{\longrightarrow} K x_{0}^{*}$ in $X^{*}$. Thus we have $\left\|x_{0}^{*}+y_{\lambda}^{*}\right\| \leqq\left\|f_{0}+y_{\lambda}^{*}\right\| \leqq\left\|y_{\lambda}^{*}\right\|+\varepsilon\left\|f_{0}\right\|=$ $K\left\|x_{0}^{*}\right\|+\varepsilon\left\|f_{0}\right\|$. By taking the weak* limit, we have

$$
\left\|x_{0}^{*}+K x_{0}^{*}\right\| \leqq \sup _{\lambda}\left\|x_{0}^{*}+y_{\lambda}^{*}\right\| \leqq K\left\|x_{0}^{*}\right\|+\varepsilon\left\|f_{0}\right\|
$$

which implies that $\left\|x_{0}^{*}\right\| \leqq \varepsilon\left\|f_{0}\right\|$ for all $\varepsilon>0$. This contradicts $x_{0}^{*} \neq 0$. Thus we have $x_{0}^{*}=0$ and $x_{0}^{* * *}=f_{0} \in X^{\perp}$.

We restate the relationships among the five classes we have defined.

$\left(L_{0}\right) \varsubsetneqq(L), \quad(L)^{*} \subset(M), \quad(M) \varsubsetneqq(P)$ and $(P) \varsubsetneqq(U)$, where $(L)^{*}=$ $\left\{X^{*} \mid X \in(L)\right\} . \quad$ Thus if a Banach space $X$ is in class $\left(L_{0}\right)$ or class $(L)$ then $X^{*}$ is the unique predual of $X^{* *}$ and if $X$ is in class $(M)$ 
or class $(P)$ then $X$ is the unique predual of $X^{*}$.

In the following separate sections, we will show that each class $\left(L_{0}\right),(L),(M)$ and $(P)$ is closed under certain operations. More examples including those showing proper inclusions among the classes will be supplied in each section.

3. Banach spaces in class $(L)$. One shows that class $\left(L_{0}\right)$ is closed under taking quotients and $c_{0}$-direct sums.

THEOREM 1.

(a) If a Banach space $X$ is in class $\left(L_{0}\right)$, then any quotient space of $X$ is also in class $\left(L_{0}\right)$.

(b) If a family of Banach spaces $X_{\lambda}(\lambda \in \Lambda)$ is in class $\left(L_{0}\right)$, then its $c_{0}$-direct sum $\left(\sum_{\lambda} X_{\lambda}\right)_{c_{0}}$ is also in class $\left(L_{0}\right)$.

Proof. (a) Suppose $Y$ is a subspace of a Banach space $X$ in class $\left(L_{0}\right)$. We have the usual identifications $(X / Y)^{*}=Y^{\perp},(X / Y)^{* *}=$ $X^{* *} / Y^{\perp \perp}$ and $(X / Y)^{* * *}=Y^{\perp \perp \perp}$. Thus the canonical decomposition $(X / Y)^{* * *}=(X / Y)^{*} \oplus(X / Y)^{\perp}$ can be identified with $Y^{\perp \perp \perp}=Y^{\perp} \oplus$ $\left(X^{\perp} \cap Y^{\perp \perp \perp}\right)$. This identification completes the proof since for $x^{*} \in$ $Y^{\perp} \subset X^{*}$ and $f \in X^{\perp} \cap Y^{\perp \perp \perp} \subset X^{\perp}$ we have from the hypothesis $\left\|x^{*}+f\right\|=\left\|x^{*}\right\|+\|f\|$.

(b) We only present the proof for the case $\Lambda$ is countable. The proof for the general case is similar. Let $X=\left(\sum_{n} X_{n}\right)_{c_{0}}$ and $X_{n} \in\left(L_{0}\right)$ $(n=1,2, \cdots)$. We claim that $\left\|x^{*}+f\right\|=\left\|x^{*}\right\|+\|f\|$ for all $x^{*} \in X^{*}$ and $f \in X^{\perp} \subset X^{* * *}$. Let $x^{*} \in X^{*}$ and $f \in X^{\perp}$. Since we can identify $X^{*}$ with $\left(\sum_{n} X_{n}^{*}\right)_{l_{1}}$, we have $x^{*}=\sum_{n} x_{n}^{*}$ with $\left\|x^{*}\right\|=\sum_{n}\left\|x_{n}^{*}\right\|$ where $x_{n}^{*} \in X_{n}^{*}(n=1,2 \cdots)$. It is sufficient to prove the identify with the assumption $x_{n}^{*}=0$ except for finitely many integers, namely, $x^{*}=$ $x_{1}^{*}+\cdots+x_{m}^{*}$ for some $m$. If $Y_{m}=\left(\sum_{n>m} X_{n}\right)_{c_{0}}$, then we have $X^{*}=$ $\left(X_{1}^{*} \oplus \cdots \oplus X_{m}^{*} \oplus Y_{m}^{*}\right)_{l_{1}}$ and $X^{* * *}=\left(X_{1}^{* * *} \oplus X_{2}^{* * *} \oplus \cdots \oplus X_{m}^{* * *} \oplus\right.$ $\left.Y_{m}^{* *}\right)_{l_{1}}$. We identify the canonical decomposition of $X^{* * *}$ with $\left(X_{1}^{*} \oplus \cdots \oplus X_{m}^{*} \oplus Y_{m}^{*}\right)_{l_{1}} \oplus\left(X_{1}^{\perp} \oplus \cdots \oplus X_{m}^{\perp} \oplus Y_{m}^{\perp}\right)_{l_{1}} \quad$ where $\quad X_{n}^{* * *}=$ $X_{n}^{*} \oplus X_{n}^{\llcorner}$for $n=1,2, \cdots, m$ and $Y_{m}^{* * *}=Y_{m}^{*} \oplus Y_{m}^{\perp}$. Using this identification, $f$ can be written as $f_{1}+\cdots+f_{m}+g$ where $f_{n} \in X_{n}^{\perp}$ $(n=1,2, \cdots, m)$ and $g \in Y_{m}^{\perp}$. Thus $x^{*}+f=x_{1}^{*}+\cdots+x_{m}^{*}+f_{1}+\cdots+$ $f_{m}+g=\left(x_{1}^{*}+f_{1}\right)+\cdots+\left(x_{m}^{*}+f_{m}\right)+g$, and we have $\left\|x^{*}+f\right\|=$ $\left\|x_{1}^{*}+f_{1}\right\|+\cdots+\left\|x_{m}^{*}+f_{m}\right\|+\|g\|$ and $\|f\|=\left\|f_{1}+\cdots+f_{m}+g\right\|=$ $\left\|f_{1}\right\|+\cdots+\left\|f_{m}\right\|+\|g\|$, because $X^{* * *}$ is the $l_{1}$-direct sum of $X_{1}^{* * *}, \cdots, X_{m}^{* * *}$ and $Y_{m}^{* * *}$. Since $\left\|x_{n}^{*}+f_{n}\right\|=\left\|x_{n}^{*}\right\|+\left\|f_{n}\right\| \quad(n=$ $1,2, \cdots, m)$, we have $\left\|x^{*}+f\right\|=\left\|x_{1}^{*}\right\|+\left\|f_{1}\right\|+\cdots+\left\|x_{m}^{*}\right\|+\left\|f_{m}\right\|+\| g \mid=$ $\left\|x_{1}^{*}\right\|+\cdots+\left\|x_{m}^{*}\right\|+\left\|f_{1}\right\|+\cdots+\left\|f_{m}\right\|+\|g\|=\left\|x^{*}\right\|+\|f\|$. This completes the proof. 
As mentioned in $\S 2, c_{0}$ is a typical example of spaces in class $\left(L_{0}\right)$. In [9], W. B. Johnson and M. Zippin considered the Banach space $C_{\infty}=\left(\sum_{n} G_{n}\right)_{c_{0}}$ where $G_{n}$ is a certain sequence of finite dimensional spaces. They point out that $C_{\infty}$ has a quotient space which fails approximation property. With Theorem 1 , one can see that $C_{\infty}$ and this quotient space are in class $\left(L_{0}\right)$. Another example of a space in class $\left(L_{0}\right)$ which is different from $c_{0}$ is as follows.

Proposition 5. The Banach space of all compact operators on a Hilbert space with the operator norm is in class $\left(L_{0}\right)$.

Proof. Let $\boldsymbol{K}(H)$ be the Banach space of all compact operators on a Hilbert space $H$ with the operator norm $\|\cdot\|$ and let $\boldsymbol{T}(H)$ be the Banach space of all operators of trace class on $H$ with the trace norm $\|\cdot\|_{\text {tr }}$. Then $\boldsymbol{T}(H)$ can be regarded as the dual space of $\boldsymbol{K}(H)$, where the duality is given by the trace, $\langle T, K\rangle=\operatorname{tr}(T K)$ for $T \in$ $\boldsymbol{T}(H)$ and $K \in \boldsymbol{K}(H) . \quad \boldsymbol{B}(H)$, the Banach space of all bounded operators on $H$ with the operator norm, can be regarded as the dual space of $\boldsymbol{T}(H)$ with the duality also given by the trace, $\langle B, T\rangle=\operatorname{tr}(T B)$ for $B \in \boldsymbol{B}(H)$ and $T \in \boldsymbol{T}(H)$. Thus the canonical decomposition $\boldsymbol{K}(H)^{* * *}=\boldsymbol{K}(H)^{*} \oplus \boldsymbol{K}(H)^{\perp}$ can be identified with $\boldsymbol{B}(H)^{*}=\boldsymbol{T}(H) \oplus$ $\boldsymbol{K}(H)^{\perp}$. We need to show that $\|T+\varphi\|=\|T\|_{\mathrm{tr}}+\|\varphi\|$ for all $T \in$ $\boldsymbol{T}(H)$ and $\varphi \in \boldsymbol{K}(H)^{\perp}$. Given $T \in \boldsymbol{T}(H), \varphi \in \boldsymbol{K}(H)^{\perp} \subset \boldsymbol{B}(H)^{*}$ and $\varepsilon>0$, we choose $K \in \boldsymbol{K}(H)$ with $\|K\|=1$ and $B \in \boldsymbol{B}(H)$ with $\|B\|=1$ such that $\langle T, K\rangle \geqq\|T\|_{\text {tr }}-\varepsilon$ and $\langle\varphi, B\rangle \geqq\|\varphi\|-\varepsilon$. Since $T$ can be approximated by a finite rank operator in $\boldsymbol{T}(H)$, there is an orthogonal projection $P$ on $H$ with a finite dimensional range such that $\|(I-P) T\|_{\mathrm{tr}}<\varepsilon$ and $\|T(I-P)\|_{\mathrm{tr}}<\varepsilon$. Setting $B_{0}=P K P+(I-P) B(I-P)$, we have $\left\|B_{0}\right\| \leqq 1$, and $\left\langle\varphi, B_{0}\right\rangle=\langle\varphi, B\rangle$ because $\varphi$ annihilates compact operators. Thus we have $\left\langle T+\varphi, B_{0}\right\rangle=\left\langle T, B_{0}\right\rangle+\left\langle\varphi, B_{0}\right\rangle=\operatorname{tr}(T P K P)+$ $\operatorname{tr}(T(I-P) B(I-P))+\left\langle\varphi, B_{0}\right\rangle=\operatorname{tr}(T K)-\operatorname{tr}(T P K(I-P))-\operatorname{tr}(T(I-$ $P) K P)-\operatorname{tr}(T(I-P) K(I-P))+\operatorname{tr}(T(I-P) B(I-P))+\langle\varphi, B\rangle$. This implies

$$
\begin{aligned}
\left|\left\langle T+\varphi, B_{0}\right\rangle\right| \geqq & |\operatorname{tr}(T K)+\langle\varphi, B\rangle| \\
& \quad-\|(I-P) T\|_{\mathrm{tr}}\|P K\|-\|T(I-P)\|_{\mathrm{tr}}\|K P\| \\
& \quad-\|T(I-P)\|_{\mathrm{tr}}\|K(I-P)\|-\|T(I-P)\|_{\mathrm{tr}}\|B\| \\
\geqq & \operatorname{tr}(T K)+\langle\varphi, B\rangle-4 \varepsilon \\
\geqq & \|T\|_{\mathrm{tr}}+\|\varphi\|-6 \varepsilon,
\end{aligned}
$$

and we have $\|T+\varphi\| \geqq\|T\|_{\mathrm{tr}}+\|\varphi\|$, which completes the proof.

It is not difficult to see that the space $c$ is not in class $\left(L_{0}\right)$, hence $C[0,1]$ is not in class $\left(L_{0}\right)$, because $c$ is a quotient space of 
$C[0,1]$. Also note that $l_{\infty}$ and $L^{\infty}[0,1]$ are not in class $\left(L_{0}\right)$. This fact will be shown in Proposition 9 in the last section of this paper.

The class $(L)$, which is larger than $\left(L_{0}\right)$, is also closed under taking of quotients and $c_{0}$-direct sums. In fact it is closed under more general direct sums:

THEOREM 2.

(a) If a Banach space $X$ is in class $(L)$, then any quotient space of $X$ is also in class $(L)$.

(b) If a family of Banach spaces $X_{\lambda}(\lambda \in \Lambda)$ is in class $(L)$ with $\sup _{\lambda} \gamma_{X_{\lambda}}(\varepsilon)<+\infty$ for all $\varepsilon>0$, then the direct sum $\left(\sum_{2} X_{\lambda}\right)_{Z}$ is in class $(L)$ for every $Z$ having property $\left(\Delta_{\infty}\right)$.

Proof. (a) As in the proof of Theorem 1, part (a), we identify the canonical decomposition $(X / Y)^{* * *}=(X / Y)^{*} \oplus(X / Y)^{\perp}$ with $Y^{\perp \perp \perp}=$ $Y^{\perp} \oplus\left(Y^{\perp} \cap Y^{\perp \perp \perp}\right)$ where $Y^{\perp} \subset X^{*} \subset X^{* * *}$ and $X^{\perp} \cap Y^{\perp \perp \perp} \subset X^{\perp} \subset X^{* * *}$. Thus $X / Y$ is in class $(L)$ with $\gamma_{X / Y}(\varepsilon)=\gamma_{X}(\varepsilon)$ for all $\varepsilon>0$.

(b) This result follows from Theorem 3 and the duality between class $(L)$ and class $(M)$. The proof will be presented in $\S 4$.

A consequence of Theorem 2 is that Banach spaces with property $\left(\Delta_{\infty}\right)$ are in class $(L)$. This fact can be generalized as follows:

Proposition 6. Suppose a Banach space $X$ satisfies

(i) There is a net of projections $P_{\lambda}(\lambda \in \Lambda)$ from $X$ onto reflexive subspaces of $X$ such that $\lim _{\lambda}\left\|P_{\lambda} x-x\right\|=0$ for all $x \in X$ and $\left\|P_{\lambda}\right\|=$ $\left\|I-P_{\lambda}\right\|=1$ for all $\lambda \in \Lambda$,

(ii) For any $\varepsilon>0$ there is a $\rho=\rho(\varepsilon)>0$ such that

$$
\|x+y\| \leqq\|x\|+\varepsilon\|y\|
$$

whenever $\|x\| \geqq \rho\|y\|, P_{\lambda} x=x$ and $P_{\lambda} y=0$ for some $\lambda \in \Lambda$. Then $X$ is in class $(L)$. In particular, every Banach space $Z$ with property $\left(\Delta_{\infty}\right)$ is in class $(L)$.

Proof. Assumption (ii) together with $\left\|P_{\lambda}\right\|=\left\|I-P_{\lambda}\right\|=1(\lambda \in \Lambda)$ implies the following dual property of (ii) on $X^{*}$; for any $\varepsilon>0$ there is a $\rho=\rho(\varepsilon)>0$ such that

$$
\left({ }^{*}\right) \quad \rho\left\|x^{*}\right\|+\left\|y^{*}\right\| \leqq(\rho+\varepsilon)\left\|x^{*}+y^{*}\right\|
$$

whenever $P_{\lambda}^{*} x^{*}=x^{*}$ and $P_{\lambda}^{*} y^{*}=0$ for some $\lambda \in \Lambda$. This can be shown in a similar fashion to the proof of part (a) of Proposition 1. Also, in a manner similar to the proof of part (b) of Proposition 1 , one can see that $\lim _{2}\left\|P_{2}^{*} x^{*}-x^{*}\right\|=0$ for all $x^{*} \in X^{*}$ follows from 
$\left(^{*}\right)$ and the assumption $\lim _{\lambda}\left\|P_{\lambda} x-x\right\|=0$ for all $x \in X$. Note that, since the range of each projection $P_{\lambda}$ is reflexive, an element $x^{* * *}$ belongs to $X^{\perp}$ in the canonical decomposition $X^{* * *}=X^{*} \oplus X^{\perp}$ if and only if $P_{\lambda}^{* * *} x^{* * *}=0$ for all $\lambda \in \Lambda$. We claim that

$$
\rho\left\|x^{*}\right\|+\|f\| \leqq(\rho+\varepsilon)\left\|x^{*}+f\right\|
$$

whenever $x^{*} \in X^{*}$ and $f \in X^{\perp}$. Since, for $x^{*} \in X^{*}, \lim _{\lambda}\left\|P_{\lambda}^{*} x^{*}-x^{*}\right\|=0$, we may assume $f \in X^{\perp}$ and $x^{*} \in X^{*}$ with $P_{\lambda_{0}^{*}}^{*} x^{*}=x^{*}$ for some $\lambda_{0} \in \Lambda$. Let $\left\{z_{\nu}^{*}\right\}$ be a net in $X^{*}$ such that $x^{*}+f=w^{*}-\lim _{\nu} z_{\nu}^{*}$ in $X^{* * *}$ and $\left\|x^{*}+f\right\|=\left\|z_{\nu}^{*}\right\|$ for all $\nu$. By setting $x_{\nu}^{*}=P_{\lambda_{0}}^{*} z_{\nu}^{*}$ and $y_{\nu}^{*}=z_{\nu}^{*}-x_{\nu}^{*}$, we see that $x^{*}=P_{\lambda_{0}}^{* * *} x^{*}=P_{\lambda_{0}}^{* *}\left(x^{*}+f\right)=w^{*}-\lim _{\nu} P_{\lambda_{0}}^{* *} z_{\nu}^{*}=w^{*}-\lim _{\nu} x_{\nu}^{*}$ in $X^{* * *}$ and $f=w^{*}-\lim _{\nu} z_{\nu}^{*}-x^{*}=w^{*}-\lim _{\nu} y_{\nu}^{*}$ in $X^{* * *}$. The inequality $(*)$ gives us

$$
\rho\left\|x_{\nu}^{*}\right\|+\left\|y_{\nu}^{*}\right\| \leqq(\rho+\varepsilon)\left\|x_{\nu}^{*}+y_{\nu}^{*}\right\|=(\rho+\varepsilon)\left\|x^{*}+f\right\|
$$

for all $\nu$, and by taking the weak* limit of a subnet, we have

$$
\rho\left\|x^{*}\right\|+\|f\| \leqq(\rho+\varepsilon)\left\|x^{*}+f\right\| .
$$

This completes the proof.

Examples of nonreflexive Banach spaces with property $\left(\Delta_{\infty}\right)$ can be given by certain Orlicz sequence spaces $h_{M}$ where $M$ is an Orlicz function; see Chapter 4 in [10] for pertinent definitions and properties. If $M$ satisfies the condition $\sup _{0<u<u_{0}} 2 M(u / 2) / M(u)=\beta<1$ for some $u_{0}>0$, then the Banach space $h_{M}$ has property $\left(\Delta_{\infty}\right)$. The proof can be seen as follows: For $\varepsilon>0$, we need to choose a $\rho=\rho(\varepsilon)>0$ such that $\left\|\left(a_{n}\right)+\left(b_{n}\right)\right\| \leqq\left\|\left(a_{n}\right)\right\|+\varepsilon$ whenever $\left\|\left(a_{n}\right)\right\| \geqq \rho,\left\|\left(b_{n}\right)\right\|=1$ and $\left(a_{n}\right) \perp\left(b_{n}\right)$. We may assume that $a_{n} \geqq 0$ and $b_{n} \geqq 0$. We can choose an integer $j$ so that $\sup _{n}\left|c_{n}\right| / 2^{j}<u_{0}$ for any $\left(c_{n}\right)$ with $\left\|\left(c_{n}\right)\right\|=$ $\sum_{n} M\left(\left|c_{n}\right|\right)=1$. Let $\left\|\left(a_{n}\right)\right\|=r$ and $k$ be an integer so that $2^{k} \leqq$ $r<2^{k+1}$. Thus we have

$$
\begin{aligned}
\sum_{n} M\left(\frac{a_{n}}{r+\varepsilon}\right)+\sum_{n} M\left(\frac{b_{n}}{r+\varepsilon}\right) \\
\quad \leqq \sum_{n} \frac{r}{r+\varepsilon} M\left(\frac{a_{n}}{r}\right)+\sum_{n} M\left(\frac{b_{n}}{r}\right) \\
\quad=\frac{r}{r+\varepsilon}+\sum_{n} M\left(\frac{b_{n}}{r}\right) \\
\quad \leqq 1-\frac{\varepsilon}{2^{k+1}+\varepsilon}+\sum_{n} M\left(\frac{b_{n}}{2^{k}}\right) \\
\quad \leqq 1-\frac{\varepsilon}{2^{k+1}+\varepsilon}+\sum_{n} \frac{\beta^{k-j}}{2^{k}} M\left(b_{n}\right) \\
\quad=1-\frac{\varepsilon}{2^{k+1}+\varepsilon}+\frac{\beta^{k-j}}{2^{k}}<1 \quad \text { if } \quad k \geqq l \text { for some } l .
\end{aligned}
$$


Thus $\left\|\left(a_{n}\right)+\left(b_{n}\right)\right\| \leqq r+\varepsilon=\left\|\left(a_{n}\right)\right\|+\varepsilon$ if $\left\|\left(a_{n}\right)\right\| \geqq \rho(\varepsilon)=2^{l}$. This completes the proof.

If $M(u)=(u+1) \ln (u+1)-u$ then the function complementary to $M$ is $M^{*}(u)=e^{u}-u-1$. Since $M^{*}$ does not satisfy the $\Delta_{2}$-condition at zero, both $h_{M}$ and $h_{M}^{*}$ are nonreflexive Banach spaces; see Proposition 4.b.2, p. 148 in [10]. Because $\lim _{u \rightarrow 0} 2 M(u / 2) / M(u)=$ $\lim _{u \rightarrow 0} 2 M^{*}(u / 2) / M^{*}(u)=1 / 2$ both $h_{M}$ and $h_{M}^{*}$ have property $\left(\Delta_{\infty}\right)$ and consequently are in class $(L)$.

By Theorem 2 the $l_{p}$-direct sum of $c_{0},\left(\sum c_{0}\right)_{l_{p}}$ with $1<p<\infty$ is in class $(L)$, although these spaces are not in class $\left(L_{0}\right)$. The canonical predual $J^{-1}$ of a James quasi-reflexive space $J$ is in class $(L)$, as will be shown in Proposition 7. Thus $\left(\sum J^{-1}\right)_{l_{p}}$ with $1<p<\infty$ and $\left(\sum J^{-1}\right)_{c_{0}}$ are in class $(L)$. Note that we will also show that successive duals of $J^{-1}$ are not in class $(L)$; see Proposition 7 .

4. Banach spaces in class $(M)$. One shows that class $(M)$ is closed under taking of certain direct sums.

THeOREM 3. If a family of Banach spaces $X_{\lambda}(\lambda \in \Lambda)$ is in class $(M)$ with $\sup _{\lambda} \rho_{X_{\lambda}}(\varepsilon)<\infty$ for all $\varepsilon>0$ then the direct sum $\left(\sum_{\lambda} X_{\lambda}\right)_{Z}$ is in class $(M)$ for every $Z$ having property $\left(\Delta_{1}\right)$.

Proof. The first step is to prove this result when $Z$ is a finite dimensional space. Assume that $X_{i}$ and $Y_{i} \subset X_{i}^{*}(i=1,2, \cdots, n)$ are given so that each $Y_{i}$ is norming over $X_{i}$, and given $\varepsilon>0$ there is a $\rho=\rho(\varepsilon)$ such that $\left\|y_{i}^{*}+f_{i}\right\| \leqq\left\|y_{i}^{*}\right\|+\varepsilon\left\|f_{i}\right\|$ whenever $y_{i}^{*} \in Y_{i}$, $f_{i} \in X_{i}^{\perp}$ and $\left\|y_{i}^{*}\right\| \geqq \rho\left\|f_{i}\right\|$ for each $i=1,2, \cdots, n$. Setting $X=$ $\left(\sum_{i=1}^{n} X_{i}\right)_{Z}$ then $X^{*}=\left(\sum_{i=1}^{n} X_{i}^{*}\right)_{Z^{*}}$ and $Y=\left(\sum_{i=1}^{n} Y_{i}\right)_{Z^{*}}$ is norming over $X$. Furthermore we have $X^{* * *}=X^{*} \oplus X^{\perp}=\left(\sum_{i=1}^{n} X_{i}^{*}\right)_{Z^{*}} \oplus\left(\sum_{i=1}^{n} X_{i}^{\perp}\right)_{Z^{*}}$.

We need to show that given $\varepsilon>0$ there is a $\tilde{\rho}=\tilde{\rho}(\varepsilon)>0$ such that $\left\|\left(\left\|y_{1}^{*}+f_{1}\right\|, \cdots,\left\|y_{n}^{*}+f_{n}\right\|\right)\right\|_{Z^{*}} \leqq\left\|\left(\left\|y_{1}^{*}\right\|, \cdots,\left\|y_{n}^{*}\right\|\right)\right\|_{Z^{*}}+\varepsilon \|\left(\left\|f_{1}\right\|, \cdots\right.$, $\left.\left\|f_{n}\right\|\right) \|_{Z^{*}}$ whenever $\left\|\cdot\left(\left\|y_{1}^{*}\right\|, \cdots,\left\|y_{n}^{*}\right\|\right)\right\|_{Z^{*}} \geqq \tilde{\rho}\left\|\left(\left\|f_{1}\right\|, \cdots,\left\|f_{n}\right\|\right)\right\|_{Z^{*}}$ and $y_{i}^{*} \in Y_{i}, f_{i} \in X_{i}^{\perp}$ for all $i=1,2, \cdots, n$. Since $Z^{*}$ has property $\left(\Delta_{\infty}\right)$ we can choose a $\bar{\rho}=\bar{\rho}(\varepsilon)$ such that

$$
\left\|\left(\xi_{i}\right)+\left(\eta_{i}\right)\right\|_{Z^{*}} \leqq\left\|\left(\xi_{i}\right)\right\|_{Z^{*}}+\frac{\varepsilon}{\rho(\varepsilon)+1}\left\|\left(\eta_{i}\right)\right\|_{Z^{*}}
$$

if $\left\|\left(\xi_{i}\right)\right\|_{Z^{*}} \geqq \bar{\rho}\left\|\left(\eta_{i}\right)\right\|_{Z^{*}}$ and $\left(\xi_{i}\right) \perp\left(\eta_{i}\right)$. Finally we let $\tilde{\rho}=\tilde{\rho}(\varepsilon)=$ $(\bar{\rho}(\varepsilon)+1)(\rho(\varepsilon)+1)$ and suppose

$$
\left\|\left(\left\|y_{1}^{*}\right\|, \cdots,\left\|y_{n}^{*}\right\|\right)\right\|_{Z^{*}} \geqq \tilde{\rho}\left\|\left(\left\|f_{i}\right\|, \cdots,\left\|f_{n}\right\|\right)\right\|_{Z^{*}} .
$$

Without loss of generality we may assume 


$$
\left\|\left(\left\|f_{1}\right\|, \cdots,\left\|f_{n}\right\|\right)\right\|_{Z^{*}}=1
$$

and also for some $k$ with $1 \leqq k \leqq n$ such that

$$
\begin{array}{ll}
\left\|y_{i}^{*}\right\| \geqq \rho\left\|f_{i}\right\| & \text { for } \quad i=1,2, \cdots, k, \\
\left\|y_{i}^{*}\right\| \leqq \rho\left\|f_{i}\right\| & \text { for } \quad i=k+1, \cdots, n .
\end{array}
$$

Thus

$$
\begin{aligned}
& \left\|\left(\left\|y_{1}^{*}+f_{1}\right\|, \cdots,\left\|y_{k}^{*}+\dot{f}_{k}\right\|, 0 \cdots, 0\right)\right\|_{Z^{*}} \\
& \geqq\left\|\left(\left\|y_{1}^{*}\right\|, \cdots,\left\|y_{k}^{*}\right\|, 0, \cdots, 0\right)\right\|_{Z^{*}}-\left\|\left(\left\|f_{1}\right\|, \cdots,\left\|f_{k}\right\|, 0, \cdots, 0\right)\right\|_{Z^{*}} \\
& \geqq\left\|\left(\left\|y_{1}^{*}\right\|, \cdots,\left\|y_{n}^{*}\right\|\right)\right\|_{Z^{*}}-\left\|\left(0, \cdots, 0,\left\|y_{k+1}^{*}\right\|, \cdots,\left\|y_{n}^{*}\right\|\right)\right\|_{Z^{*}}-1 \\
& \geqq \tilde{\rho}-\rho\left\|\left(0, \cdots, 0,\left\|f_{k+1}\right\|, \cdots,\left\|f_{n}\right\|\right)\right\|_{Z^{*}}-1 \\
& \geqq \tilde{\rho}-(\rho+1)=\bar{\rho}(\rho+1), \quad \text { and } \\
& \left\|\left(0, \cdots, 0,\left\|y_{k+1}^{*}+f_{k+1}\right\|, \cdots,\left\|y_{n}^{*}+f_{n}\right\|\right)\right\|_{Z^{*}} \\
& \quad \leqq(\rho+1)\left\|\left(0, \cdots, 0,\left\|f_{k+1}\right\|, \cdots,\left\|f_{n}\right\|\right)\right\|_{Z^{*}} \leqq \rho+1
\end{aligned}
$$

Thus we have

$$
\begin{aligned}
&\left\|\left(\left\|y_{1}^{*}+f_{1}\right\|, \cdots,\left\|y_{k}^{*}+f_{k}\right\|, 0, \cdots, 0\right)\right\|_{Z^{*}} \\
& \geqq \bar{\rho}\left\|\left(0, \cdots, 0,\left\|y_{k+1}^{*}+f_{k+1}\right\|, \cdots,\left\|y_{n}^{*}+f_{n}\right\|\right)\right\|_{Z^{*}} .
\end{aligned}
$$

Hence, by our choice of $\bar{\rho}$ we have

$$
\begin{aligned}
\|\left(\| y_{1}^{*}+\right. & \left.f_{1}\|, \cdots,\| y_{n}^{*}+f_{n} \|\right) \|_{z^{*}} \\
\leqq & \left\|\left(\left\|y_{1}^{*}+f_{1}\right\|, \cdots,\left\|y_{k}^{*}+f_{k}\right\|, 0, \cdots, 0\right)\right\|_{Z^{*}} \\
& \quad+\frac{\varepsilon}{\rho+1}\left\|\left(0, \cdots, 0,\left\|y_{k+1}^{*}+f_{k+1}\right\|, \cdots,\left\|y_{n}^{*}+f_{n}\right\|\right)\right\|_{Z^{*}} \\
\leqq & \left\|\left(\left\|y_{1}^{*}\right\|+\varepsilon\left\|f_{1}\right\|, \cdots,\left\|y_{k}^{*}\right\|+\varepsilon\left\|f_{k}\right\|, 0, \cdots, 0\right)\right\|_{Z^{*}}+\varepsilon \\
\leqq & \left\|\left(\left\|y_{1}^{*}\right\|, \cdots,\left\|y_{k}^{*}\right\|, 0, \cdots, 0\right)\right\|_{Z^{*}}+\varepsilon+\varepsilon \\
\leqq & \left\|\left(\left\|y_{1}^{*}\right\|, \cdots,\left\|y_{n}^{*}\right\|\right)\right\|_{Z^{*}}+2 \varepsilon
\end{aligned}
$$

We turn our attention to the general case. Assume that $X_{\lambda}$ and $Y_{\lambda} \subset X_{\lambda}^{*}(\lambda \in \Lambda)$ are given such that each $Y_{\lambda}$ is norming over $X_{\lambda}$. If $X=\left(\sum_{\lambda} X_{\lambda}\right)_{Z}$, then $X^{*}=\left(\sum_{\lambda} X_{\lambda}^{*}\right)_{Z^{*}}$. Let $Y$ be the norm closed linear span of $\left\{Y_{\lambda}\right\}_{\lambda \in A}$ in $\left(\sum_{\lambda} X_{\lambda}^{*}\right)_{Z^{*}}$, then $Y$ is a norming subspace of $X^{*}$ over $X$. For a subset $F$ of $\Lambda$ we write $X_{F}=\left(\sum_{\lambda \in F} X_{\lambda}\right)_{\left.z\right|_{F}}$ and thus $\left(X_{F}\right)^{*}=X_{F}^{*}=\left(\sum_{\lambda \in F} X_{\lambda}^{*}\right)_{\left.Z^{*}\right|_{F}}$. Furthermore the following identifications can be made: $X=X_{F} \oplus X_{A \backslash F}, X^{*}=X_{F}^{*} \oplus X_{\Lambda \backslash F}^{*}$ and $X^{* * *}=$ $\left(X_{F}\right)^{* * *} \oplus\left(X_{\Lambda \backslash F}\right)^{* * *}$. Furthermore the canonical decompositions $\left(X_{F}\right)^{* * *}=X_{F}^{*} \oplus\left(X_{F}\right)^{\perp}$ and $\left(X_{\Lambda \backslash F}\right)^{* * *}=X_{\Lambda \backslash F}^{*} \oplus\left(X_{\Lambda \backslash F}\right)^{\perp}$ lead to the canonical decomposition of $X^{* * *} ; X^{* * *}=X^{*} \oplus X^{\perp}=\left(X_{F}^{*} \oplus X_{A F}^{*}\right) \oplus$ $\left(\left(X_{F}\right)^{\perp} \oplus\left(X_{\Lambda \backslash F}\right)^{\perp}\right)$. Since $Z$ has property $\left(\Delta_{1}\right), Z^{*}$ has property $\left(\Delta_{\infty}\right)$ implying that for any $\varepsilon>0$ there is a $\bar{\rho}=\bar{\rho}(\varepsilon)>0$ such that $\left\|x_{1}^{*}+x_{2}^{*}\right\| \leqq\left\|x_{1}^{*}\right\|+\varepsilon\left\|x_{2}^{*}\right\|$ whenever $x_{1}^{*} \in X_{F}^{*}, x_{2}^{*} \in X_{\Lambda N^{*}}^{*}$ and $\left\|x_{1}^{*}\right\| \geqq$ 
$\bar{\rho}\left\|x_{2}^{*}\right\|$. Since the unit ball of $X_{F}^{*}\left(X_{\Lambda \backslash F}^{*}\right)$ is weak* dense in the unit ball of $\left(X_{F}\right)^{* * *}\left(\left(X_{\Lambda \backslash F}\right)^{* * *}\right)$, this same inequality holds for the decomposition $X^{* * *}=\left(X_{F}\right)^{* * *} \oplus\left(X_{\Lambda \backslash F}\right)^{* * *}$;

$$
\left\|x_{1}^{* * *}+x_{2}^{* * *}\right\| \leqq\left\|x_{1}^{* * *}\right\|+\varepsilon\left\|x_{2}^{* * *}\right\|
$$

whenever $x_{1}^{* * *} \in\left(X_{F}\right)^{* * *}, x_{2}^{* * *} \in\left(X_{\Lambda \backslash F}\right)^{* * *}$ and $\left\|x_{1}^{* * *}\right\| \geqq \bar{\rho}\left\|x_{2}^{* * *}\right\|$. For a finite subset $F$ of $\Lambda$ we have shown that for any $\varepsilon>0$ there is a $\tilde{\rho}=\tilde{\rho}(\varepsilon)>0$ such that

$$
\left\|y^{*}+f\right\| \leqq\left\|y^{*}\right\|+\varepsilon\|f\|
$$

whenever $y^{*} \in X_{F}^{*} \cap Y, f \in\left(X_{F}\right)^{\perp}$ and $\left\|y^{*}\right\| \geqq \tilde{\rho}\|f\|$. Note that $\bar{\rho}$ and $\tilde{\rho}$ are independent of our choice of a finite subset $F$ of $\Lambda$.

Given $\varepsilon$ with $0<\varepsilon<1$, we set $\tilde{\tilde{\rho}}=\overline{\tilde{\rho}}(\varepsilon)=\operatorname{Max}\{\tilde{\rho}(\varepsilon), 1+\bar{\rho}(\varepsilon)(\varepsilon+1)\}$ and we claim that $\left\|y^{*}+f\right\| \leqq\left\|y^{*}\right\|+4 \varepsilon\|f\|$ whenever $y^{*} \in Y, f \in X^{\perp}$ and $\left\|y^{*}\right\|>\tilde{\tilde{\rho}}\|f\|$. Without loss of generality we assume $\|f\|=1$. We choose a finite subset $F \subset \Lambda$ so that $y^{*}=y_{1}^{*}+y_{2}^{*}, y_{1}^{*} \in X_{F}^{*} \cap Y$, $y_{2}^{*} \in X_{i \backslash F}^{*} \cap Y,\left\|y_{1}^{*}\right\|>\tilde{\tilde{\rho}}$ and $\left\|y_{2}^{*}\right\| \leqq \varepsilon$. Then we have $f=f_{1}+f_{2}$ with $f_{1} \in\left(X_{F}\right)^{\perp}$ and $f_{2} \in\left(X_{\Lambda \backslash F}\right)^{\perp}$. Note that $\left\|f_{1}\right\|,\left\|f_{2}\right\| \leqq\|f\|=1$, because the projections from $X^{* * *}$ onto $\left(X_{F}\right)^{* * *}$ and $\left(X_{\Lambda \backslash F}\right)^{* * *}$ are of norm 1. Since $\left\|y_{1}^{*}+f_{1}\right\|>\overline{\tilde{\rho}}-1 \geqq \bar{\rho}(\varepsilon)(\varepsilon+1) \geqq \bar{\rho}(\varepsilon)\left\|y_{2}^{*}+f_{2}\right\|$, we have $\left\|y^{*}+f\right\|=\left\|y_{1}^{*}+f_{1}+y_{2}^{*}+f_{2}\right\| \leqq\left\|y_{1}^{*}+f_{1}\right\|+\varepsilon\left\|y_{2}^{*}+f_{2}\right\| \leqq$ $\left\|y_{1}^{*}+f_{1}\right\|+\varepsilon(\varepsilon+1) \leqq\left\|y_{1}^{*}+f_{1}\right\|+2 \varepsilon$. Furthermore since $\left\|y_{1}^{*}\right\| \geqq \tilde{\tilde{\rho}} \geqq$ $\tilde{\rho}(\varepsilon)\left\|f_{1}\right\|$ we have $\left\|y_{1}^{*}+f_{1}\right\| \leqq\left\|y_{1}^{*}\right\|+\varepsilon\left\|f_{1}\right\| \leqq\left\|y_{1}^{*}\right\|+\varepsilon \leqq\left\|y_{1}^{*}+y_{2}^{*}\right\|+2 \varepsilon$. Thus $\left\|y^{*}+f\right\| \leqq\left\|y^{*}\right\|+4 \varepsilon$ and the proof of the theorem is complete.

The proof of part (b) of Theorem 2 is completed by observing that (i) if $Z$ has property $\left(\Delta_{\infty}\right)$ then the basis of $Z$ is shrinking (see Proposition 1, (b)), (ii) the norm closed linear span of $\left\{X_{\lambda}\right\}_{\lambda \in A}$ in $\left(\sum_{\lambda} X_{*}^{* *}\right)_{Z * *}$ is $\left(\sum_{2} X_{\lambda}\right)_{Z}=X$ and (iii) Proposition 3 on the duality between the class $(L)$ and the class $(M)$.

In $\S 6$, we will show that a James quasi-reflexive space $J$ is in class $(L)^{*} \subset(M)$. Thus for $1 \leqq p<\infty,\left(\sum J\right)_{l_{p}}$ is in class $(M)$. We do not have any example of a Banach space in class $(M)$ which is not in class $(L)^{*}$. Note that Theorem 3 will not give us such an example because if a family of Banach spaces $X_{\lambda}(\lambda \in A)$ is in class $(L)$ with $\sup _{\lambda_{2}} \gamma_{X_{\lambda}}(\varepsilon)<+\infty$ for all $\varepsilon>0$ then $\left(\sum_{\lambda} X_{\lambda}^{*}\right)_{Z}$ is in class $(L)^{*}$ for every $Z$ with property $\left(\Delta_{1}\right)$.

5. Banach spaces in class $(P)$. One shows that class $(P)$ is closed under taking of more general direct sums than class $(M)$.

THeOREM 4. If a family of Banach spaces $X_{\lambda}(\lambda \in \Lambda)$ is in class $(P)$ then the direct sum $\left(\sum_{\lambda} X_{\lambda}\right)_{Z}$ is in class $(P)$ for every $Z$ which 
has property $\left(\Delta_{1}\right)$ or is reflexive.

Proof. (a) $Z$ has property $\left(\Delta_{1}\right)$. We will use the notations and the canonical identifications used in the proof of Theorem 3 (We replace $F$ by a single point $\left.\lambda_{0}\right)$. If $X=\left(\sum_{\lambda} X_{\lambda}\right)_{Z}$ is not in class $(P)$, there is a nonzero $z_{0}^{*} \in X^{*}$ and $f_{0} \in X^{\perp}$ so that

$$
\left\|f_{0}+x^{*}\right\| \geqq\left\|z_{0}^{*}+x^{*}\right\| \quad \text { for all } x^{*} \in X^{*} \text {. }
$$

Choose $\lambda_{0}$ so that $z_{0}^{*}=z_{1}^{*}+z_{2}^{*}$ with $z_{1}^{*} \neq 0, z_{1}^{*} \in X_{\lambda_{0}}^{*}$ and $z_{2}^{*} \in X_{\Lambda \backslash\left\{\lambda_{0}\right.}^{*}$. Furthermore we have $f_{0}=f_{1}+f_{2}$ with $f_{1} \in\left(X_{\lambda_{0}}\right)^{\perp}$ and $f_{2} \in\left(X_{\Lambda \backslash \backslash \lambda_{0} !}\right)^{\perp}$.

Since $Z^{*}$ has property $\left(\Delta_{\infty}\right)$ we have the following property of the decomposition $X^{*}=X_{\lambda_{0}^{*}}^{*} \oplus X_{i \backslash\left\{\lambda_{0}\right\}}^{*}$ for any $\varepsilon>0$ there is a $\bar{\rho}=$ $\bar{\rho}(\varepsilon)>0$ such that $\left\|x_{1}^{*}+x_{2}^{*}\right\| \leqq\left\|x_{1}^{*}\right\|+\varepsilon\left\|x_{2}^{*}\right\|$ whenever $x_{1}^{*} \in X_{\lambda_{0}}^{*}$, $x_{2}^{*} \in X_{A \backslash\left\{\lambda_{0}\right\}}^{*}$ and $\left\|x_{1}^{*}\right\| \geqq \bar{\rho}\left\|x_{2}^{*}\right\|$. Since the unit ball of $X_{\lambda}^{*}\left(X_{\left.A \backslash \lambda_{0}\right\}}^{*}\right)$ is weak ${ }^{*}$ dense in the unit ball of $X_{\lambda_{0}^{* *}}^{* *}\left(\left(X_{\left.\Lambda \backslash \lambda_{0}\right\}}\right)^{* * *}\right)$, this same inequality holds for the decomposition $X^{* * *}=X_{\lambda_{0}}^{* *} \oplus\left(X_{\Lambda \backslash\left\{\lambda_{0}\right\}}\right)^{* * *}$;

$$
\begin{aligned}
\left\|x_{1}^{* * *}+x_{2}^{* * *}\right\| \leqq & \left\|x_{1}^{* * *}\right\|+\varepsilon\left\|x_{2}^{* * *}\right\| \\
& \text { whenever } x_{1}^{* * *} \in X_{\lambda_{0}}^{* * *}, \quad x_{2} \in\left(X_{\Lambda \backslash\left(\lambda_{0}\right)}\right)^{* * *}
\end{aligned}
$$

and $\left\|x_{1}^{* * *}\right\| \geqq \bar{\rho}\left\|x_{2}^{* * *}\right\|$.

Since $X_{\lambda_{0}}$ is in class $(P)$ and $z_{1}^{*} \neq 0$ is in $X_{\lambda_{0}}^{*}$, there is a $\delta>0$ so that

$$
E=\left\{y_{1}^{*} \in X_{\lambda_{0}}^{*}\|\| f_{1}+y_{1}^{*}\|+\delta<\| z_{1}^{*}+y_{1}^{*} \|\right\}
$$

is not empty. If $y_{1}^{*}$ is in $E$, then one can see that $(n+1)\left(z_{1}^{*}+y_{1}^{*}\right)-y_{1}^{*}$, is also in $E$ for all $n=1,2, \cdots$. Thus $E$ is unbounded.

If $f_{2}=0$, we let $y_{1}^{*}$ be any element in $E$ and if $f_{2} \neq 0$ we choose $y_{1}^{*} \in E$ with sufficiently large norm so that

$$
\left\|f_{1}+y_{1}^{*}\right\|>\bar{\rho}\left(\frac{\delta}{\left\|f_{2}\right\|}\right)\left\|f_{2}\right\| .
$$

If $f_{2} \neq 0$, then, by inequality (1)

$$
\begin{aligned}
\left\|f_{0}+y_{1}^{*}\right\| & =\left\|f_{1}+y_{1}^{*}+f_{2}\right\|=\left\|f_{1}+y_{1}^{*}\right\|+\frac{\delta}{\left\|f_{2}\right\|}\left\|f_{2}\right\| \\
& =\left\|f_{1}+y_{1}^{*}\right\|+\delta<\left\|z_{1}^{*}+y_{1}^{*}\right\| .
\end{aligned}
$$

Thus we have $\left\|f_{0}+y_{1}^{*}\right\|<\left\|z_{1}^{*}+y_{1}^{*}\right\|$. This inequality clearly holds when $f_{2}=0$ and $y_{1}^{*}$ is in $E$. We arrive at the contradiction:

$$
\left\|f_{0}+y_{1}^{*}\right\|<\left\|z_{1}^{*}+y_{1}^{*}\right\| \leqq\left\|z_{0}^{*}+y_{1}^{*}\right\| \text {. }
$$

The last inequality depends on the fact that the projections associated with the decomposition $X^{*}=X_{\lambda_{0}}^{*} \oplus X_{\Lambda \backslash\left\{\lambda_{0}\right\}}^{*}$ are of norm 1 . 
(b) $Z$ is reflexive. If $X=\left(\sum_{\lambda} X_{\lambda}\right)_{z}$ then one can identify $X^{*}$ with $\left(\sum_{2} X_{i}^{*}\right)_{Z^{*}}$ and $X^{* * *}$ with $\left(\sum_{\lambda} X_{i}^{* * *}\right)_{Z^{*}}$. We have $X^{* * *}=$ $X^{*} \oplus X^{\perp}=\left(\sum_{\lambda} X_{\lambda}^{*}\right)_{Z^{*}} \oplus\left(\sum_{\lambda} X_{\lambda}^{\perp}\right)_{Z^{*}}$. We need to show that if given $z^{*}=\left(z_{i}^{*}\right)$ in $X^{*}$ and $f=\left(f_{\lambda}\right)$ in $X^{\perp}$ so that $\left\|f+x^{*}\right\| \geqq\left\|z^{*}+x^{*}\right\|$ for all $x^{*}$ in $X^{*}$, then $z^{*}=0$. If $z^{*}$ is not zero then we choose $\lambda_{1} \in \Lambda$ so that $z_{\lambda_{1}}^{*} \neq 0$ and $\delta>0$ so that

$$
E=\left\{x_{1}^{*} \in X_{\lambda_{1}}^{*} \mid\left\|f_{\lambda_{1}}+x_{1}^{*}\right\|+\delta<\left\|z_{\lambda_{1}}^{*}+x_{1}^{*}\right\|\right\}
$$

is not empty. As observed in (a), $E$ is unbounded in $X_{\lambda_{1}}^{*}$. Finally we choose a finite subset $F$ of $A$ so that $\lambda_{1}$ is in $F$ and

$$
\left\|\left.\left(f_{2}\right)\right|_{\Lambda F F}\right\|<\varepsilon \delta
$$

where $\varepsilon>0$ will be chosen later.

We denote $F=\left\{\lambda_{1}, \lambda_{2}, \cdots, \lambda_{n}\right\}, z_{i_{i}}^{*}=z_{i}^{*}$ and $f_{\lambda_{2}}=f_{i}$ for $i=1,2, \cdots, n$. We may assume that there is a $k$ with $1 \leqq k \leqq n$ so that $z_{\imath}^{*} \neq 0$ for $i=1,2, \cdots, k$ and $z_{\imath}^{*}=0$ for $i=k+1, \cdots, n$. Since each $X_{i}$ is in class $(P)$ we choose $y_{i}^{*} \in X_{i_{2}}^{*} i=2, \cdots, n$ so that

$$
\begin{aligned}
& \left\|f_{i}+y_{i}^{*}\right\| \leqq\left\|z_{i}^{*}+y_{i}^{*}\right\| \quad \text { for } i=2, \cdots, k, \\
& \left\|f_{i}+y_{i}^{*}\right\| \leqq \delta^{\prime}+\left\|y_{i}^{*}\right\| \quad \text { for } i=k+1, \cdots, n
\end{aligned}
$$

where $\hat{o}^{\prime}=\varepsilon \delta /\|(\underbrace{0, \cdots, 0}_{k}, 1, \cdots, 1)\|_{Z^{*} F_{F}}$. For any $x_{1}^{*} \in X_{\lambda_{1}}^{*}$ if we set $x^{*}=\left(x_{1}^{*}, y_{2}^{*}, \cdots, y_{n}^{*}\right)$ we have the following inequality:

$$
\begin{aligned}
\left\|f+x^{*}\right\| \leqq & \left\|\left(f_{1}, \cdots, f_{n}\right)+x^{*}\right\|+\varepsilon \delta \\
= & \left\|\left(\left\|f_{1}+x_{1}^{*}\right\|,\left\|f_{2}+y_{2}^{*}\right\|, \cdots,\left\|f_{n}+y_{n}^{*}\right\|\right)\right\|_{Z^{*} F_{F}}+\varepsilon \delta \\
\leqq & \|\left(\left\|f_{1}+x_{1}^{*}\right\|,\left\|z_{2}^{*}+y_{2}^{*}\right\|, \cdots,\left\|z_{k}^{*}+y_{k}^{*}\right\|,\right. \\
& \left.\delta^{\prime}+\left\|y_{k+1}^{*}\right\|, \cdots, \delta^{\prime}+\left\|y_{n}^{*}\right\|\right) \|_{Z^{*} \mid F}+\varepsilon \delta \\
\leqq & \left\|\left(\left\|f_{1}+x_{1}^{*}\right\|,\left\|z_{2}^{*}+y_{2}^{*}\right\|, \cdots,\left\|z_{n}^{*}+y_{n}^{*}\right\|\right)\right\|_{Z^{*} F^{*}}+2 \varepsilon \delta \delta .
\end{aligned}
$$

We claim that

$$
\begin{aligned}
\left\|\left(\left\|f_{1}+x_{1}^{*}\right\|,\left\|z_{2}^{*}+y_{2}^{*}\right\|, \cdots,\left\|z_{n}^{*}+y_{n}^{*}\right\|\right)\right\|_{\left.Z^{*}\right|_{F}} \\
+2 \varepsilon \delta<\|\left(\left\|z_{1}^{*}+x_{1}^{*}\right\|, \cdots,\left\|z_{n}^{*}+y_{n}^{*}\right\|\right)_{\left.Z^{*}\right|_{F}}
\end{aligned}
$$

if $x_{1}^{*}$ is in $E$ with sufficiently large norm and $2 \varepsilon<(1-\varepsilon)\left\|e_{\lambda_{1}}^{*}\right\|_{Z^{*}}$ where $e_{\lambda_{1}}^{*}$ is the element of $Z^{*}$ with the $\lambda_{1}$-coordinate $=1$ and $\lambda$ coordinate $=0$ for all $\lambda \neq \lambda_{1}$. This can be seen as follows. The function $\varphi(t)=\left\|\left(t,\left\|z_{2}^{*}+y_{2}^{*}\right\|, \cdots,\left\|z_{n}^{*}+y_{n}^{*}\right\|\right)\right\|_{\left.Z^{*}\right|_{F}}$ is a convex function for $t \geqq 0$. Thus we have $\left(\varphi\left(t_{2}\right)-\varphi\left(t_{1}\right)\right) /\left(t_{2}-t_{1}\right) \geqq\left(\varphi\left(t_{1}\right)-\varphi(0)\right) /\left(t_{1}-0\right)>$ $(1-\varepsilon)\|(1,0, \cdots, 0)\|_{\left.Z^{*}\right|_{F}} \geqq 2 \varepsilon$ for sufficiently large $t_{1}$ and $t_{2}>t_{1}$. This implies that

$$
\varphi\left(t_{2}\right) \geqq\left(t_{2}-t_{1}\right) 2 \varepsilon+\varphi\left(t_{1}\right)
$$


The result follows by putting $t_{1}=\left\|f_{1}+x_{1}^{*}\right\|$ and $t_{2}=\left\|z_{1}^{*}+z_{1}^{*}\right\|$ for an element $x_{1}^{*} \in E$ with sufficiently large norm. We have shown that $\left\|f+x^{*}\right\|<\left\|\left(z_{1}^{*}, \cdots, z_{n}^{*}\right)+x^{*}\right\| \leqq\left\|z^{*}+x^{*}\right\|$. This contradicts our assumption and completes the proof.

An example of a Banach space in class $(P)$ is $X=\left(\sum X_{p_{n}}\right)_{l_{p}}$ where $X_{p_{n}}=\left(\sum l_{1}\right)_{l_{p}}$ and $1 \leqq p, p_{n}<\infty$. Note that if $p_{n} \rightarrow \infty$ then $X$ is not in class $(L)^{*}$. In a previous paper [2] we have shown that the James quasi-reflexive space $J$ and all its successive duals are in $(P)$. In the next section we will show that all of the successive duals of $J$ are not in class $(M)$.

6. Some examples.

(i) The James quasi-reflexive Banach spaces. The James space $(J,\|\cdot\|)$ is defined in [7] to be the space consisting of all complex sequences $x=(x(n))$ such that

$$
\|x\|=\sup \left(\sum_{j=1}^{k}\left|\sum_{n \in I_{j}} x(n)\right|^{2}\right)^{1 / 2}<+\infty
$$

where the supremum is taken over all choices of disjoint finite intervals $I_{1}, I_{2}, \cdots, I_{k}$ of positive integers. James [8] also introduced an equivalent norm $\|\cdot \cdot\| \mid$ on $J$ for which $(J,\|\cdot \cdot\| \mid)$ is isometrically isomorphic to $(J,|| \cdot \mid \|)^{* *}$ (see [2], Theorem 3).

In this section, $J$ will always stand for $(J,\|\cdot\|)$. The space $J$ has the natural normalized basis $\left\{e_{n}\right\}$; for every $x \in J$ we have $x=$ $\sum_{n=1}^{\infty} x(n) e_{n}$, where $e_{n}=\left(e_{n}(j)\right)=\left(\delta_{n, j}\right)$ for $n, j=1,2, \cdots$. Let $\left\{e_{n}^{*}\right\}$ be the biorthogonal sequence with respect to $\left\{e_{n}\right\}$ and let $Y$ be the closed linear span of $\left\{e_{n}^{*}\right\}$. Since $\left\{e_{n}\right\}$ is a boundedly complete monotone basis of $J, J$ is isometrically isomorphic to the dual $Y^{*}$ of $Y$ by the canonical mapping (see p. 9 of [10]). We introduce the linear functionals $\varphi_{n}$ on $J$ by $\varphi_{n}(x)=\sum_{j=n}^{\infty} x(j)$ for $x \in J$ and $n=1,2, \cdots$. As proved in [7], $\left(\varphi_{n}\right\}$ forms a normalized basis of $J^{*}$ and we have

$$
J^{*}=\left[\varphi_{1}\right] \oplus Y .
$$

We define $f_{2} \in J^{* *}$ as follows; $f_{2}\left(\varphi_{1}\right)=1$ and $f_{2}$ annihilates $Y$. Then we have $J^{* *}=\left[f_{2}\right] \oplus J$. More generally, denote $J^{n}(n=0,1,2, \cdots)$ as the $n$ 'th dual of $J$, then we have

$$
\begin{aligned}
& J=J^{0} \subset J^{2} \subset \cdots \subset J^{2 n} \subset \cdots, \\
& J^{*}=J^{1} \subset J^{3} \subset \cdots \subset J^{2 n+1} \subset \cdots .
\end{aligned}
$$

For $n \geqq 3, f_{n} \in J^{n}$ is defined as follows: $f_{n}\left(f_{n-1}\right)=1$ and $f_{n}$ annihilates $J^{n-1}$. Thus the one dimensional space $\left[f_{n}\right]$ is a norm 1 complement of $J^{n-2}$ in $J^{n}$ and we have

$$
J^{n}=\left[f_{n}\right] \oplus J^{n-2} \quad \text { for } \quad n=2,3, \cdots .
$$


For even $n \geqq 2,\left\{f_{n}, f_{n-2}, \cdots, f_{4}, f_{2}, e_{1}, e_{2}, \cdots\right\}$ forms a basis of $J^{n}$ and for odd $n \geqq 3,\left\{f_{n}, f_{n-2}, \cdots, f_{5}, f_{3}, \varphi_{1}, e_{1}^{*}, e_{2}^{*}, \cdots\right\}$ forms a basis of $J^{n}$.

In Theorems 1 and 2 of [2], we have shown that $J^{n}, n=$ $-1,0,1, \cdots$ are in class $(P)$, where $J^{-1}=Y$ is the unique predual of $J=J^{0}$, we also showed that $(J, \||\cdot| \mid)$ and $(J, \||\cdot| \mid)^{*}$ are in class $(P)$.

Proposition 7.

(a) $J^{-1}$ is in class $(L)$ and $J^{n}$ is not in class $(L)$ if $n \neq-1$,

(b) $J$ is in class $(M)$ and $J^{n}$ is not in class $(M)$ if $n \neq 0$.

Proof. (a-1) $J^{-1}$ is in class $(L)$. Proposition 3 implies that it is sufficient to show that in the canonical decomposition $J^{* * *}=$ $J^{*} \oplus J^{\perp}=J^{*} \oplus\left[f_{3}\right]$, for any $\varepsilon>0$ there is a $\rho=\rho(\varepsilon)>0$ such that $\left\|x^{*}+f_{3}\right\| \leqq\left\|x^{*}\right\|+\varepsilon$ whenever $x^{*} \in Y=J^{-1}$ and $\left\|x^{*}\right\| \geqq \rho$. If $x^{*}=$ $\sum_{k=1}^{n} \beta_{k} e_{k}^{*}$, then because $f_{3}=w^{*}-\lim _{l} \varphi_{l}$ in $J^{* * *}$, we have

$$
\left\|x^{*}+f_{3}\right\| \leqq \sup _{l>n}\left\|\sum_{k=1}^{n} \beta_{k} e_{k}^{*}+\varphi_{l}\right\| .
$$

If $x=\sum_{k=1}^{\infty} \alpha_{k} e_{k}$ is in $J$, then $x=x_{1}+x_{2}$ where $x_{1}=\sum_{k=1}^{n} \alpha_{k} e_{k}$ and $x_{2}=\sum_{k=n+1}^{\infty} \alpha_{k} e_{k}$. Thus $\left|\left(x^{*}+\varphi_{l}\right)(x)\right|=\left|x^{*}\left(x_{1}\right)+\varphi_{l}\left(x_{2}\right)\right| \leqq\left\|x^{*}\right\|\left\|x_{1}\right\|+$ $\left\|\varphi_{l}\right\|\left\|x_{2}\right\| \leqq\left(\left\|x^{*}\right\|^{2}+\left\|\varphi_{l}\right\|^{2}\right)^{1 / 2}\left(\left\|x_{1}\right\|^{2}+\left\|x_{2}\right\|^{2}\right)^{1 / 2} \leqq\left(\left\|x^{*}\right\|^{2}+1\right)^{1 / 2}\|x\|$. Hence $\left\|x^{*}+\varphi_{l}\right\| \leqq\left(\left\|x^{*}\right\|^{2}+1\right)^{1 / 2}$ if $l>n$, and we have $\left\|x^{*}+f_{*}\right\| \leqq\left(\left\|x^{*}\right\|^{2}+1\right)^{1 / 2}$. This inequality holds for all $x^{*}$ in $J^{-1}$ by the continuity of the norm. One can see that, for any $\varepsilon>0$, if $x^{*}$ is in $J^{-1}$ and $\left\|x^{*}\right\|$ is sufficiently large then

$$
\left\|x^{*}+f_{3}\right\| \leqq\left(\left\|x^{*}\right\|^{2}+1\right)^{1 / 2} \leqq\left\|x^{*}\right\|+\varepsilon .
$$

(a-2) $J^{n}$ is not in class $(L)$ if $n \neq-1$. If $J^{n}$ were in class $(L)$ then for a given $\varepsilon>0$ there is a $\delta=\delta(\varepsilon)>0$ such that if $\left\|f_{n+3}+x_{n+1}\right\|=1$ and $1-\left\|x_{n+1}\right\|<\delta$ for $x_{n+1} \in J^{n+1}$ then $\left\|f_{n+3}\right\|<\varepsilon$. For $n \geqq 0$, we will choose $x_{n+1}$ in $J^{n+1}$ so that $\left\|f_{n+3}+x_{n+1}\right\|=\left\|x_{n+1}\right\|=1$. Thus we have a contradition, $1 \leqq\left\|f_{n+3}\right\|<\varepsilon$. Let $x_{2}=-f_{2}$ and $x_{2 n+2}=$ $-f_{2 n+2}-x_{2 n}$ for $n \geqq 1$. Let $x_{1}=-\varphi_{1}$ and $x_{2 n+1}=-f_{2 n+1}-x_{2 n-1}$ for $n \geqq 1$. Then we have $f_{n+3}+x_{n+1}=-x_{n+3}$ for all $n \geqq 0$. We complete the proof of this part by showing that $\left\|x_{n}\right\|=1$ for all $n \geqq 1$. In Lemma 2 of [2], we showed $\left\|f_{2}\right\|=1$, thus we have $\left\|x_{2}\right\|=1$. Assume $\left\|x_{2 n}\right\|=1$, then $1=\left\|x_{2 n}\right\| \leqq\left\|f_{2 n+2}+x_{2 n}\right\|=\left\|x_{2 n+2}\right\|$. Since $f_{2 n+2}=w^{*}-$ $\lim _{k}\left(-x_{2 n}+(-1)^{n} e_{k}\right)$ in $J^{2 n+2}$ (see [2], Lemma 1, (a)), we have $\left\|x_{2 n+2}\right\|=$ $\left\|f_{2 n+2}+x_{2 n}\right\| \leqq \sup _{k}\left\|-x_{2 n}+(-1)^{n} e_{k}+x_{2 n}\right\|=\sup _{k}\left\|(-1)^{n} e_{k}\right\|=1$. The proof is completed for even $n$. For odd $n$, the proof is completed in a similar fashion by using the facts the $f_{2 n+1}=w^{*}-\lim _{k}\left(x_{2 n-1}+\right.$ $(-1)^{n} \varphi_{1}+(-1)^{n+1} \varphi_{k}$ ) in $J^{2 n+1}$ (see [2], Lemma 1, (b)) and $\left\|\varphi_{k}-\varphi_{1}\right\|=1$ for all $k>1$. Thus we have shown that $J^{n}$ is not in class $(L)$ for 
all $n \geqq 0$.

(b-1) $\quad J^{-1}=Y$ is not in class $(M)$. This can be seen by observing that $J^{* *}=Y^{* * *}=Y^{*} \oplus Y^{\perp}=J \oplus\left[f_{2}\right]$ and showing that if $Z$ is a subspace of $J$ which norms $Y$ then $Z=Y$. Since $Z$ norms $Y$, $Z^{\perp} \cap Y=\{0\}$. If $Z \neq J$ then $Z^{\perp} \neq\{0\}$ and $J^{*}=Z^{\perp} \oplus Y$ since $\operatorname{dim} J^{*} / Y=1$. For any $x^{*} \in Z^{\perp}$ and $y^{*} \in Y,\left\|x^{*}+y^{*}\right\| \geqq \sup \left\{\mid\left(x^{*}+\right.\right.$ $\left.\left.y^{*}\right)(x)|| x \in Z,\|x\| \leqq 1\right\}=\sup \left\{\mid y^{*}(x)\|x \in Z\|, x \| \leqq 1\right\}=\left\|y^{*}\right\|$. Thus $Y$ has a norm 1 complement in $J^{*}$. This contradicts the fact that $Y$ is not norm 1 complemented in $J^{*}$ (see the proof of Theorems 1,2 ) of [2]. Thus $J$ has no proper subspace of $J=Y^{*}$ which norms $Y$. This implies that $Y$ is not in class $(M)$. See the remark after the definition of class $(M)$ in $\S 2$.

(b-2) $J$ is in class $(M)$. From $(a-1), J^{-1}$ is in class $(L)$. Hence $J=\left(J^{-1}\right)^{*}$ is in class $(L)^{*}$ which is contained in class $(M)$.

(b-3) $J^{n}$ is not in class $(M)$ if $n>0$. This can be seen by observing that $J^{n+3}=J^{n+1} \oplus\left(J^{n}\right)^{\perp}=J^{n+1} \oplus\left[f_{n+3}\right]$ and showing that if $Z$ is a subspace of $J^{n+1}$ which norms $J^{n}$ then $Z=J^{n+1}$ or $Z=J^{n-1}$. Thus if $J^{n}$ were in class $(M)$ then $Z=J^{n-1}$ and Proposition 3 says that $J^{n-1}$ is in class $(L)$. This contradicts part (a-2). Since $Z$ norms $J^{n}, Z^{\perp} \cap J^{n}=\{0\}$. If $Z \neq J^{n+1}$, then we have $J^{n+2}=Z^{\perp} \oplus J^{n}$. Again one sees that $Z^{\perp}$ is a norm 1 complement of $J^{n}$ in $J^{n+2}$. We have shown in [2] that $\left[f_{n+2}\right]$ is the only norm 1 complement of $J^{n}$ in $J^{n+2}$; see the proof of Theorem 1, (1) of [2]. Hence $Z^{\perp}=\left[f_{n+2}\right]$, so $Z=$ $\left(Z^{\perp}\right)^{\top}=\left[f_{n+2}\right]$, so $Z=\left(Z^{\perp}\right)^{\top}=\left[f_{n+2}\right]^{\top}=J^{n-1}$. The proof is complete.

The following proposition is proven in a manner similar to Proposition 7 and we omit the proof.

Proposition 8. $(J,\||\cdot|\|)$ and $(J,\||\cdot|\|)^{*}$ are neither in class $(L)$ nor in class $(M)$.

Note that the Banach spaces $(J,\|\cdot\|)^{n}$ for $n>0,(J,\||\cdot|\|)$ and $(J,|| \cdot|| \mid)^{*}$ are in class $(P)$ but they are not in class $(M)$.

(ii) The Banach spaces $L^{\infty}$ and $H^{\infty}$. As noted in the introduction, the Banach spaces $L^{\infty}$ and $H^{\infty}$ on the unit circle have unique preduals, namely, $L^{1}$ and $L^{1} / H_{0}^{1}$. This fact is actually proved by showing that $L^{1}$ and $L^{1} / H_{0}^{1}$ are in class $(U)$; see [1], [6] and [12]. The purpose of this part is to point out the following:

Proposition 9. (a) $L^{1}$ and $L^{1} / H_{0}^{1}$ are not in class $(P)$.

(b) $L^{1}(\mu)$, where $\mu$ is a $\sigma$-finite measure, is in class $(P)$ if and only if $\mu$ is purely atomic.

(c) The dual of $C(\Omega)$, where $\Omega$ is a compact Housdorff space, is in class $(P)$ if and only if $\Omega$ has no perfect compact subset. 
Proof. (a-1) $L^{1}$ is not in class $(P)$. The following proof is a modification of our proof suggested by $P$. Wojtaszczyk which also enables one to prove $L^{1} / H_{0}^{1}$ is not in class $(P)$.

We have the usual identification of $L^{\infty}=C(\Omega)$, where $\Omega$ is a compact Housdorff space and $\left(L^{\infty}\right)^{*}=M(\Omega)$, the space of all finite Randon measures on $\Omega$. Furthermore $M(\Omega)=M_{a} \bigoplus_{l_{1}} M_{s}$ where $M_{a}\left(M_{s}\right)$ is the set of all measures absolutely continuous (singular) with respect to the measure on $\Omega$ associated with Lebesque measure on the unit circle, and $L^{1}$ is identified with $M_{a}$. The canonical decomposition $\left(L^{1}\right)^{* * *}=\left(L^{1}\right)^{*} \oplus\left(L^{1}\right)^{\perp}$ can be identified with $M(\Omega)^{*}=C(\Omega) \oplus M_{a}^{\perp}$. We claim that for any $f_{0}$ in $C(\Omega)$ there is a $\varphi$ in $M_{a}^{\perp}$ such that

$$
\|\varphi+f\| \geqq\left\|f_{0}+f\right\| \text { for all } f \in C(\Omega) \text {. }
$$

Let $P$ be the projection onto $M_{s}$ associated with the decomposition $M(\Omega)=M_{a} \oplus M_{s}$, then $P^{*}$ is a norm 1 projection from $M(\Omega)^{*}$ onto $M_{a}^{\perp}$. For given $f_{0}$ in $C(\Omega) \subset M(\Omega)^{*}$ let $\phi=P^{*} f_{0}$. Then for any $f$ in $C(\Omega)$

$$
\begin{aligned}
\left\|f_{0}+f\right\| & =\sup \left\{\left|f_{0}(w)+f(w)\right| \| w \in \Omega\right\} \\
& \leqq \sup \left\{\left\|\mu\left(f_{0}+f\right)\right\| \mu \in M_{s},\|\mu\| \leqq 1\right\} \\
& \leqq \sup \left\{\mid P \mu\left(f_{0}+f\right)\|\mu \in M,\| \mu \| \leqq 1\right\} \\
& =\left\|P^{*} f_{0}+P^{*} f\right\|=\left\|P^{*}\left(P^{*} f_{0}+f\right)\right\| \leqq\left\|P^{*} f_{0}+f\right\| \\
& =\|\varphi+f\| .
\end{aligned}
$$

This completes the proof of part $(a-1)$.

(a-2) $L^{1} / H_{0}^{1}$ is not in class $(P)$. This is proved in a manner similar to part $(\mathrm{a}-1)$ by using the decomposition $\left(L^{1} / H_{0}^{1}\right)^{* *}=$ $L^{1} / H_{0}^{1} \bigoplus_{l_{1}} M_{s} / M_{s} \cap\left(H^{\infty}\right)^{\perp}$ (this decomposition follows from the abstract F. and M. Riesz Theorem; see theorem 7.6 on p. 44 in [4]) and the projection $P$ of $\left(L^{1} / H_{0}^{1}\right)^{* *}$ onto $M_{s} / M_{s} \cap\left(H^{\infty}\right)^{\perp}$ associated with this decomposition.

(b) If $\mu$ is purely atomic then $L^{1}(\mu)$ is isometrically isomorphic to $l_{1}$ which is in class $(P)$ since $c_{0}$ is in class $\left(L_{0}\right)$. If $\mu$ has no atoms then a trivial modification of the proof of part (a-1) shows that $L^{1}(\mu)$ is not in class $(P)$. The proof is completed by showing that if a Banach space $X$ is the $l_{1}$-direct sum of Banach spaces $Y$ and $Z$ and $Y$ is not in class $(P)$ then $X$ is not in class $(P)$. Since $X^{* * *}=$ $Y^{* * *} \bigoplus_{l_{\infty}} Z^{* * *}=\left(Y^{*}+Y^{\perp}\right) \bigoplus_{l_{\infty}}\left(Z^{*} \oplus Z^{\perp}\right)$, the canonical decomposition of $X^{* * *}$ is $X^{*} \oplus X^{\perp}=\left(Y^{*} \bigoplus_{l_{\infty}} Z^{*}\right) \oplus\left(Y^{\perp} \bigoplus_{l_{\infty}} Z^{\perp}\right)$. If $Y$ is not in class $(P)$, there is a nonzero $y_{0}^{*}$ in $Y^{*}$ and $f_{0}$ in $Y^{\perp}$ so that $\left\|f_{0}+y^{*}\right\| \geqq\left\|y_{0}^{*}+y^{*}\right\|$ for all $y^{*} \in Y^{*}$. Thus we have for $y^{*}$ in $Y^{*}$ and $z^{*}$ in $Z^{*},\left\|f_{0}+y^{*}+z^{*}\right\|=\operatorname{Max}\left\{\left\|f_{0}+y^{*}\right\|,\left\|z^{*}\right\|\right\} \geqq$ $\operatorname{Max}\left\{\left\|y_{0}^{*}+y^{*}\right\|,\left\|z^{*}\right\|\right\}=\left\|y_{0}^{*}+y^{*}+z^{*}\right\|$ and we have $\left\|f_{0}+x^{*}\right\| \geqq$ $\left\|y_{0}^{*}+x^{*}\right\|$ for all $x^{*}$ in $X^{*}$ which says that $X$ is not in class $(P)$. 
(c) The proof of this part follows from the argument given in part (b) and the fact that the dual of $C(\Omega)$ contains a non-atomic measure if and only if $\Omega$ contains a perfect compact subset.

(iii) Remark. A von Neumann algebra has a unique predual which is in class $(U)$. This was pointed out in the introduction. It is known that the second dual of a von Neumann algebra is a von Neumann algebra; see Theorem 1.17.2, p. 43 of [11]. This raises the question if a Banach space $X$ has a unique predual, does $X^{* *}$ have a unique predual? This problem remains open. We do not even know if $X$ is in class $(U)$ must $X^{* *}$ be in class $(U)$. However, all other classes we have defined are not invariant under taking of the second dual. Examples: $c_{0}$ is in class $\left(L_{0}\right)$ and $(L)$ but $c_{v}^{* *}=l_{\infty}$ is not in class $(L)$ because $\left(l_{\infty}\right)^{*}$ is not in class $(P)$; see Proposition 9, (c). The James quasi-reflexive space $J$ is in class $(L)^{*}$ and in class $(M)$ but $J^{* *}$ is not in class $(M)$; see Proposition $8 . l_{1}$ is in class $(P)$ but $l_{1}^{* * *}=\left(l_{\infty}\right)^{*}$ is not in class $(P)$.

Addendum: In a recent paper by G. Godefroy (Espaces de Banach: Existence et unicité de certains préduax, Ann. Inst. Fourier, Grenoble, 28, no. 3 (1978), 87-105) several different conditions are presented; each of which imply that a Banach spaces is in the class $(U)$ defined in this paper. Many of the examples of Banach spaces which delineate the classes $\left(L_{0}\right),(L),(M)$ and $(P)$ mentioned in this paper satisfy one of these conditions. However, it is an open question as to the relationship between Godefroy's result and these classes.

\section{REFERENCES}

1. T. Ando, On the predual of $H^{\infty}$, Comment Math. Prace Mat, Special Issue (1978), $33-40$.

2. L. Brown and T. Ito, Isometric preduals of James spaces, Canad. J. Math., 32 (1980), $59-69$.

3. J. Dixmier, Sur un Theorem de Banach, Duke Math. J., 15 (1948), 1057-1071.

4. T. W. Gamelin, Uniform Algebras, Prentice-Hall, Inc., U.S.A., 1969.

5. A. Grothendieck, Une Caracterisation Vectorielle-Metrique de Espaces $L^{1}$, Canad. Math. J., 7 (1955), 552-561.

6. T. Ito, On Banach spaces with unique isometric preduals, Michigan Math. J., (1977), 321-324.

7. R.C. James, Bases and reflexivity of Banach spaces, Ann. of Math., 52 (1950), 518-527.

8. - A non-reflexive Banach space isometric with its second conjugate space, Proc, Nat. Acad. Sci. U.S.A., 37 (1951), 174-177.

9. W. B. Johnson and M. Zippin, Subspaces and Quotient Spaces of $\left(\sum G_{n}\right) l_{p}$ and $\left(\sum G_{n}\right)_{c_{0}}$, Israel J. Math., 19 (1974), 50-55.

10. J. Lindenstrauss and L. Tzafriri, Classical Banach Spaces I; Sequence Spaces, 
Springer-Verlag, Berlin, 1977.

11. S. Sakai, $C^{*}$-Algebras and $W^{*}$-Algebras, Springer-Verlag, Berlin, 1971.

12. P. Wojtaszczyk, On projections in spaces of bounded analytic functions, with applications, Studia Math., 65 (1979), 147-173.

Received June 14, 1979. Research partially supported by N.S.F. Grant No. MCS 78-02402.

Wayne State University

Detroit, MI 48202 
